\title{
Molecular Mechanism of Polycation-Induced Pore Formation in Biomembranes
}

\author{
Neha Awasthi, ${ }^{*}+\odot$ Wojciech Kopec, ${ }^{\ddagger}, \nabla$ Natalia Wilkosz, ${ }^{\ddagger}$ Dorota Jamróz, ${ }^{\ddagger}$ Jochen S. Hub, ${ }^{\dagger}$ \\ Maria Zatorska, Rafał Petka, Maria Nowakowska, and Mariusz Kepczynski*, ${ }^{*}+0$ \\ ${ }^{\dagger}$ Institute for Microbiology and Genetics, Georg-August-Universität, Justus-von-Liebig Weg 11, 37077 Göttingen, Germany \\ ${ }^{\ddagger}$ Faculty of Chemistry, Jagiellonian University, Gronostajowa 2, 30-387 Kraków, Poland
}

\section{Supporting Information}

\begin{abstract}
Polycations are an attractive class of macromolecules with promising applications as drug/gene carriers and biocides. The chemical structure and concentration of a polycation determine its interaction with cellular membranes and, hence, are crucial parameters for designing efficient nontoxic polycations. However, the interaction of polycations with biomembranes at the molecular level and the corresponding free-energy landscape is not well understood. In this work, we investigate the molecular mechanism of interaction between a strong polycation substituted with alkyl moieties and zwitterionic membranes via long-time-scale allatom molecular dynamics simulations and free-energy
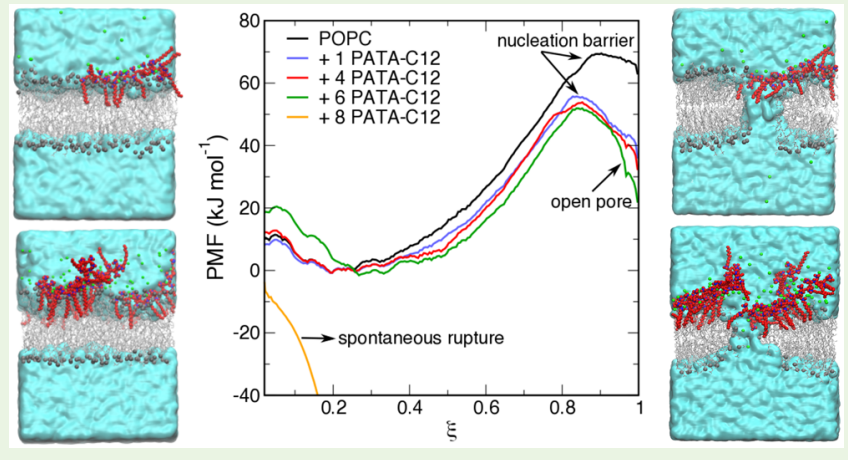
calculations combined with Langmuir monolayer, atomic force microscopy, and calcein-release experimental measurements. We found that the membrane activity of the polycation and its ability to induce pores in the membranes can be attributed to the polycation-induced changes in the bilayer organization, such as reduced membrane thickness, increased disorder of the acyl chains, reduced packing, and electrostatic field gradients between membrane leaflets. These changes facilitate the penetration of water into the membrane and the formation of aqueous defects/pores. The calculated free-energy profiles indicate that the polycation lowers the nucleation barrier for pore opening and the free energy for pore formation in a concentration-dependent manner. Above the critical coverage of the membrane, the polycation nucleates spontaneous pores in zwitterionic membranes. Our work demonstrates the potential of combining enhanced sampling methods in MD simulations with experiments for a quantitative description of various events in the polycation-membrane interaction cycle, such as strong adsorption on the membrane due to hydrophobic and electrostatic interactions, and pore formation.
\end{abstract}

KEYWORDS: polyelectrolytes, pore formation, lipid membranes, MD simulations, free energy, Langmuir monolayers

\section{INTRODUCTION}

Synthetic polycations are used in many biomedical applications, such as drug delivery vectors, transfectants, and biocides, ${ }^{1}$ and they are considered attractive model systems for cell-penetrating peptides. Understanding the interaction of various polycations with lipid membranes is crucial for designing novel polycations with improved physicochemical properties and safety, and it is tailored to the specific application. ${ }^{2-5}$ Polycations exhibit wide range of interactions with zwitterionic membranes that are used as models for mammalian cellular membranes; adsorption on the bilayer surface, ${ }^{6}$ insertion into the hydrophobic bilayer core, ${ }^{3}$ pore formation, ${ }^{7,8}$ and disruption of the bilayer into mixed polymer-lipid micelles or other aggregates. ${ }^{9,10}$ Such polycation-membrane interactions are primarily determined by the polycation architecture and its concentration. It has been shown that the presence of hydrophobic side chains in the structure of polycations plays an important role in the polymer-membrane interactions. ${ }^{11-15}$ In our previous work, we addressed the effect of hydrophobic substitution of strong polycations on the polymer-zwitterionic membrane interactions, and especially the possibility of pore formation in lipid bilayers. ${ }^{16}$ However, the molecular mechanism of interaction between polycations and lipid membranes remains poorly understood, and in particular, the free-energy landscape of transmembrane pore formation remains unknown.

The polymer-membrane interaction at the molecular scale can be investigated by performing atomistic molecular dynamics (MD) simulations. ${ }^{17}$ However, modeling the complete sequence of events in polycation-bilayer systems, namely, adsorption from solution on the bilayer, followed by insertion into the bilayer interior and eventual pore formation, is currently beyond the time scales accessible through equilibrium atomistic

Received: November 28, 2018

Accepted: December 3, 2018

Published: December 3, 2018 
MD simulations. ${ }^{18}$ Although the initial steps, i.e., polymer adsorption and insertion, have been simulated with equilibrium $\mathrm{MD},{ }^{14,16}$ special enhanced sampling techniques such as the umbrella sampling method are required to model pore formation in the presence of polycations. In umbrella sampling, a biasing potential applied along a chosen reaction coordinate drives the system from one thermodynamic state to another (such as from a no-pore state to an open-pore state). ${ }^{19}$ Hence, given a reasonable choice of reaction coordinate, the system is able to access otherwise inaccessible regions of the configuration space, including transition states, and cross energy barriers. A complete set of umbrella sampling calculation requires a series of separate simulations, called umbrella windows, biasing the system around a different configuration along the selected reaction coordinate. The separate simulation windows are combined and unbiased using methods such as the weighted histogram analysis method (WHAM) ${ }^{20}$ to obtain the potential of mean force (PMF) between the two states. ${ }^{21}$ Importantly, such a PMF along the selected reaction coordinate is also referred as the free-energy profile. ${ }^{22}$ PMFs for pore formation in pure lipid bilayers have been previously calculated using various reaction coordinates. ${ }^{23-26}$ However, the addition of membraneactive peptides or polycations introduces additional complexity in quantitative understanding molecular details of pore formation in lipid bilayers.

In this work, we focus on the molecular mechanism of pore formation in zwitterionic bilayers induced by the presence of hydrophobically modified polycations. To this end, the interaction of a strong polycation substituted with alkyl moieties, poly(allyltrimethylammonium chloride-co-allyl- $N, N$-dimethyl$\mathrm{N}$-dodecylammonium chloride (PATA-C12), with 1-palmitoyl-2-oleoyl-sn-glycero-3-phosphocholine (POPC) membrane was investigated using computational and experimental methods. We performed two sets of MD simulations: (i) multiple independent long (microsecond)-time-scale simulations of systems with different concentrations of PATA-C12 initially placed in the aqueous phase, to observe spontaneous pore formation, if any, and (ii) umbrella sampling simulations to calculate the free energy for pore formation $\left(\Delta G_{\text {pore }}\right)$, as a function of PATA-C12 concentrations, using a recently developed method for efficient calculations of $\Delta G_{\text {pore }}{ }^{19,25} \mathrm{MD}$ simulations were complemented by Langmuir monolayer and calcein-release experiments, and atomic force microscopy (AFM) visualization. The Langmuir monolayer experiments were used to estimate the impact of PATA-C12 on the organization of lipid molecules in the monomolecular film. It is known that the monolayer properties in the range of surface pressures of $30-35 \mathrm{mN} / \mathrm{m}$, such as the area per lipid, correspond to those of the bilayer. ${ }^{27}$ The permeability of the POPC membranes in the absence and presence of PATA-C12 molecules was estimated via the leakage of a fluorescent dye encapsulated in the POPC liposomes. The direct AFM visualizations were used to observe the effect of PATA-C12 on the lipid membrane.

We found that PATA-C12 is membrane-active and induces pores by increasing the membrane fluidity and decreasing membrane thickness, thus considerably reducing the values of $\Delta G_{\text {pore }}$, as well as the nucleation barrier to open pores in the POPC bilayer. Furthermore, PATA-C12 facilitates pore formation in zwitterionic membranes in a concentrationdependent manner. Taken together, our work provides a detailed molecular and thermodynamic view on polycationinduced pore formation in zwitterionic membranes.

\section{EXPERIMENTAL SECTION}

Materials. 1-Palmitoyl-2-oleoyl-sn-glycero-3-phosphocholine (POPC, $\geq 99.0 \%$ ), calcein, and chloroform were obtained from Sigma-Aldrich and used as received. Poly(allyltrimethylammonium chloride-co-allyl- $\mathrm{N}, \mathrm{N}$-dimethyl- $\mathrm{N}$-dodecylammonium chloride) (PATA-C12) was prepared according to the procedure reported previously. ${ }^{14,16}$ Millipore-quality water was used in all experiments.

Preparation of Calcein-Loaded (CL) Liposomes. Small unilamellar vesicles (SUVs) were prepared by extrusion as described previously. ${ }^{28}$ Briefly, POPC (12.5 mg) was dissolved in chloroform $(300 \mu \mathrm{L})$ in a volumetric flask. The solvent was evaporated under a gentle stream of nitrogen to form a dry lipid film. Calcein $(60 \mathrm{mM})$ dissolved in PBS was added until the desired lipid concentration was reached (usually $2.5 \mathrm{mg} / \mathrm{mL}$ ), and the sample was vortex-mixed for 5 $\mathrm{min}$. The resulting multilamellar vesicle dispersion was subjected to five freeze-thaw cycles from liquid-nitrogen temperature to $60^{\circ} \mathrm{C}$ and then extruded six times through a $100 \mathrm{~nm}$ membrane filter, using a gaspressure extruder. A commercial kit from Sigma-Aldrich was used to determine the phospholipid concentration.

Calcein-Release Studies. Fluorescence spectra were recorded using a PerkinElmer LSD 50B spectrofluorimeter equipped with a thermostated cuvette holder. The appropriate amount of the polymer solution was promptly introduced to the CL liposomes. Changes in the fluorescence intensity caused by the calcein release from the vesicles were monitored. The experiments were conducted at $25^{\circ} \mathrm{C}$. Complete dye release was achieved by adding $30 \mu \mathrm{L}$ of a $15 \%$ Triton X-100 solution. The corresponding fluorescence intensity was used as $100 \%$ leakage. The amount of calcein released after time $t, \operatorname{RF}(t)$, was calculated using the equation

$$
\operatorname{RF}(t)=100\left(\frac{I_{\mathrm{t}}-I_{0}}{I_{\text {max }}-I_{0}}\right)
$$

where $I_{0}, I_{\mathrm{t}}$ and $I_{\max }$ are fluorescence intensities measured without the polymer, at time $t$ after the polymer introduction, and after the Triton $\mathrm{X}-100$ addition, respectively. The experiments were repeated three times.

Monolayer Experiments. Surface pressure $(\pi)$-area $(A)$ isotherms were recorded using a KSV NIMA 5000 Langmuir trough (KSV Instruments Ltd., Helsinki, Finland), as described previously. ${ }^{29}$ Briefly, POPC was dissolved in chloroform to form a spreading solution $\left(c_{\mathrm{POPC}}\right.$ $=0.465 \mathrm{mg} / \mathrm{mL}$ ). The solution was deposited onto the subphase containing different concentrations of PATA-C12 $(0,0.001 \mathrm{~g} / \mathrm{L}$ or 0.01 $\mathrm{g} / \mathrm{L}$ in $1 \mathrm{mM} \mathrm{NaCl})$. The monolayers were left for $5 \mathrm{~min}$ and then compressed at a barrier speed of $10 \mathrm{~mm} / \mathrm{min}$. The surface pressure was measured to a resolution of $\pm 0.1 \mathrm{mN} / \mathrm{m}$ using a Wilhelmy plate made of filter paper (ashless Whatman Chr1) connected to an electrobalance.

Analysis of Isotherms. The basic properties of Langmuir monolayers concerning their molecular organization and the miscibility between their components can be drawn from the course and location of the recorded $\pi-A$ isotherms. To verify the state of the investigated films and possible phase transitions and to obtain information on the molecular ordering of molecules in the monolayer, the compression modulus $\left(C_{\mathrm{S}}\right.$, defined as the reciprocal of the monolayer compressibility) was calculated according to the following equation:

$$
C_{\mathrm{S}}^{-1}=-A \frac{\mathrm{d} \pi}{\mathrm{d} A}
$$

where $A$ is the area per molecule at the surface pressure of $\pi$.

Atomic Force Microscopy (AFM). Visualization of lipid bilayer surfaces was performed using a Dimension Icon AFM microscope (Bruker, Madison, WI, USA) working in the PeakForce tapping mode in liquid. The instrument was equipped with a standard V-shaped silicon nitride cantilever with a spring constant of $\sim 0.7 \mathrm{~N} \mathrm{~m}^{-1}$ and a tip radius of $\sim 20 \mathrm{~nm}$.

To prepare supported lipid bilayers, a $100 \mu \mathrm{L}$ drop of POPC liposome dispersion $(0.5 \mathrm{mg} / \mathrm{mL})$ in $0.1 \mathrm{M} \mathrm{NaCl}$ was deposited on a cleaned silicon surface (single-side polished silicon, Cemat Silicon). After $30 \mathrm{~min}$, excess lipids were gently rinsed with water and a $0.5 \mathrm{~mL}$ 
droplet of $0.1 \mathrm{M} \mathrm{NaCl}$ solution was deposited. The sample was imaged with AFM to confirm the formation of a stable continuous lipid bilayer. When the bilayer was obtained, the PATA-C12 solution in $0.1 \mathrm{M} \mathrm{NaCl}$ was introduced by a syringe into the sample volume (the final PATAC12 concentration was $\sim 0.005 \mathrm{mg} / \mathrm{mL}$ ) and the lipid bilayer was visualized with AFM.

\section{MOLECULAR DYNAMICS (MD) SIMULATIONS}

System Preparation. A bilayer containing 288 POPC molecules was taken from our previous study, ${ }^{16}$ hydrated with $\sim 30000$ water molecules, and equilibrated for 50 ns. An oligomer consisting of 30 allyltrimethylammonium units and substituted with 10 dodecyl side groups was used as a model of PATA-C12 (referred to as "PATA-C12 molecule", shown in Figure 1), as described previously. ${ }^{16}$ To study the influence of

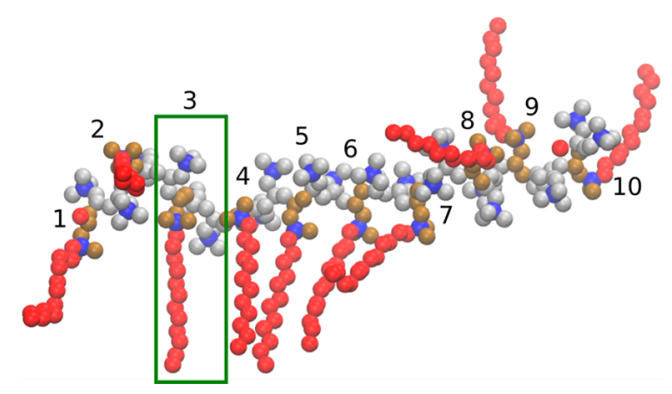

Figure 1. Single PATA-C12 molecule consisting of 30 allyltrimethylammonium units and substituted with 10 dodecyl side chains. Green rectangle highlights a single unit in the polymer. Nitrogen atoms are shown as blue spheres, atoms in the PN2 residue as ochre spheres, atoms in the PN3 residue as silver spheres, and atoms in the LAU and $\mathrm{MET}$ residues as red spheres. Atom names, types, and associated partial charges are presented in Figure S2 and Table S1 in the Supporting Information.

the density of the PATA-C12 repeating units per POPC molecules $\left(d_{\text {unit/POPC }}\right)$ on the membrane properties, we prepared five systems containing the POPC bilayer and $N=1,2,4,6$, and 8 molecules of PATA-C12. Table 1 presents a summary of the simulated systems. All N PATA-C12 molecules were placed near the upper leaflet (referred to as "outer leaflet"; the lower leaflet is called the "inner leaflet") of the equilibrated POPC bilayer and overlapping water molecules were removed. No lipids were removed from the bilayer. We note that this approach allows the polycations to self-embed, and, hence, avoids artifacts in MD simulations arising due to initial configurations where the strongly charged polycation is artificially embedded into the hydrophobic bilayer core. ${ }^{16,18}$ In addition, our simulation setup closely mimics the experimental setup, in which the polymer is added to the liposomes such that only the outer lipid layer is exposed to the polymer. Energy minimization was performed after the addition of counterions, and all the systems were equilibrated for $50 \mathrm{~ns}$.

The equilibrated systems were used for two sets of simulations: long equilibrium simulations and umbrella sampling simulations.

(a) Long equilibrium simulations were performed to analyze the influence of different polymer coverage on the POPC bilayer. To this end, three sets of independent, 1- $\mu$ s-long production simulations were performed for each $d_{\text {unit/POPC }}$ (systems M1-M8, Table 1). For reference, the pure POPC bilayer was simulated for $500 \mathrm{~ns}$ (system POPC). Membrane properties such as bilayer thickness, area per lipid (APL), and
Table 1. Summary of the Simulated Systems ${ }^{a}$

\begin{tabular}{lcccc}
\multicolumn{1}{c}{ system } & $\begin{array}{c}\text { number of } \\
\text { PATA-C12 } \\
\text { molecules, } N\end{array}$ & $\begin{array}{c}\text { number of } \\
\text { POPC } \\
\text { molecules }\end{array}$ & $d_{\text {unit/POPC }}{ }^{b}$ & $\begin{array}{c}\text { simulation } \\
\text { time }[\mathrm{ns}]\end{array}$ \\
POPC & 0 & 288 & 0 & $3 \times 500$ \\
M1 & 1 & 288 & 0.10 & $3 \times 1000$ \\
M2 & 2 & 288 & 0.21 & $3 \times 1000$ \\
M4 & 4 & 288 & 0.42 & $3 \times 1000$ \\
M6 & 6 & 288 & 0.62 & $3 \times 1000$ \\
M8 & 8 & 288 & 0.83 & $3 \times 40$ \\
UmbrellaM1 & 1 & 288 & 0.10 & $30 \times 300$, \\
& & & & $24 \times 50$ \\
UmbrellaM4 & 4 & 288 & 0.42 & $30 \times 300$ \\
& & & & $24 \times 50$ \\
UmbrellaM6 & 6 & 288 & 0.62 & $30 \times 300$, \\
& & & & $24 \times 50$ \\
UmbrellaM8 & 8 & 288 & 0.83 & $30 \times 40,24$ \\
& & & & $\times 50$
\end{tabular}

${ }^{a}$ The table indicates the number of PATA-C12 $(N)$ and POPC molecules (288) in a given system, the number of repetitions for equilibrium simulations ( 3 for POPC, M1-M8), number of umbrella windows (30 and 24 for UmbrellaM1-UmbrellaM8 for the waterdensity and slice coordinate, respectively), and the corresponding simulation times (300 and $50 \mathrm{~ns}$ for the water-density and slice coordinates, respectively). "Umbrella" refers to umbrella simulations for the potential of mean force calculations (see text for details). ${ }^{b} d_{\text {unit/POPC }}$ is the number of the PATA-C12 repeating units per POPC molecule in the system.

acyl chain deuterium order parameters $\left(\left|S_{\mathrm{CD}}\right|\right)$ were analyzed for each system. The values of $\left|S_{\mathrm{CD}}\right|$ were calculated as previously described, ${ }^{29}$ while the bilayer thickness was determined as the average distance between the phosphate groups of the upper and lower leaflets. The APL was calculated for the outer and inner membrane leaflets using the FATSLiM program (only lipid headgroups were included in the calculations, to ensure consistency with the monolayer experiments), ${ }^{30}$ and the average APL was estimated. For both APL and bilayer thickness, average values were estimated using block averages over the entire trajectories, and standard errors were calculated. Finally, the $\left|S_{\mathrm{CD}}\right|$ values were calculated for the palmitoyl $(s n 1)$ chain of POPC for all lipids in the bilayer (average $\mid S_{\mathrm{CD}} \mathrm{l}$ ) and also for lipids in each leaflet (leaflet $\mid S_{\mathrm{CD}} \mathrm{I}$ ) from the three equilibrium MD trajectories. To elucidate the insertion of the polycation molecules in the membrane, mass density profiles for the nitrogen atoms $(\mathrm{N})$ and the terminal $\mathrm{CH}_{3}$ groups of dodecyl chains of the polymer (CCL), as well as for POPC double bonds $(\mathrm{C}=\mathrm{C})$ and phosphate groups $(\mathrm{P} 8)$, chloride ions $\left(\mathrm{Cl}^{-}\right)$, and water oxygen atoms (OW) were calculated for all the M1-M8 systems, averaged over the entire trajectory, and for all $N$ molecules in a given system. Calculated mass densities from the trajectories were averaged and were scaled by 0.1 for OW and by 10 for CCL and N. To investigate the mobility of polymers adsorbed on the membrane surface, we calculated lateral diffusion constants $(D)$ for the polycation molecules and POPC lipid molecules, as previously described..$^{31}$ Mean square displacement as a function of time $((\operatorname{MSD}(t))$ was calculated for each polycation molecule in a given system and for all three trajectories. Center of mass motions of each leaflet were removed separately. $D$ was obtained by fitting a straight line to the linear region of $\operatorname{MSD}(t)$ plots for the $120 \mathrm{~ns}$ (Figure $S 1$ in the Supporting Information). The final $D$ values are averages over three trajectories of a given system, and also average over $N$ polycations molecules in a given system. The same procedure 


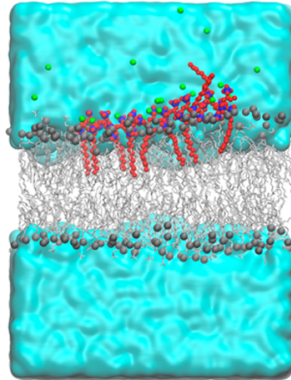

$\mathrm{t}=100 \mathrm{~ns}$

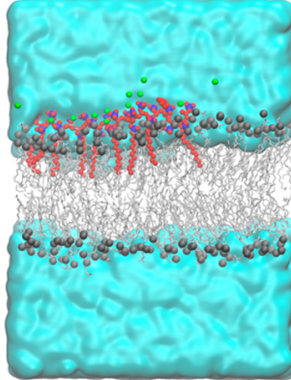

400 ns

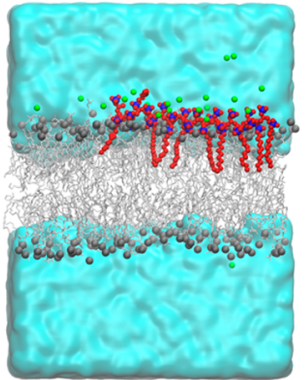

800 ns

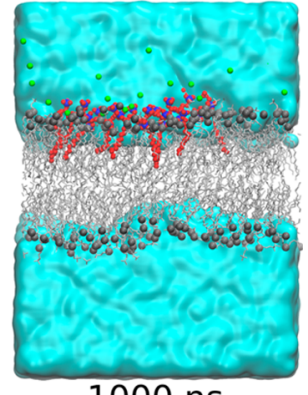

$1000 \mathrm{~ns}$

Figure 2. Snapshots of system M1 (with a single PATA-C12 molecule) taken at $t=100,400,800$, and 1000 ns of the simulations. The PATA-C12 molecule is shown as red spheres with the nitrogen atoms in blue. Lipids are shown as gray sticks, and their phosphate groups are shown as gray spheres. Chloride ions are represented as green spheres.

was repeated to calculate $D$ for POPC molecules to quantify the average lipid mobility in the absence and presence of polycations. Adhesion of positively charged polymers on a single membrane leaflet can lead to a difference in the electrostatic potential between the membrane leaflets. Accordingly, the electrostatic potentials along the bilayer normal were calculated for the POPC and M1-M6 systems using the last 400 ns of the trajectories, and for the M8 system using 40 ns of the trajectory.

(b) Umbrella sampling simulations were performed to calculate the potential of mean force (PMF) to quantitatively estimate the influence of $d_{\text {unit/POPC }}$ on pore formation. PMFs were calculated along two different reaction coordinates.

Water-Density Coordinate. PMFs were calculated along a water-density reaction coordinate, ${ }^{19,25}$ where a membranespanning cylinder is filled with water molecules $\left(N_{\mathrm{w}}\right)$. Initial frames for umbrella sampling were taken from a slow-growth pore-opening simulation. Starting from the equilibrium state, the system was pulled up to 130 water molecules in the membrane-spanning cylinder $\left(N_{\mathrm{w}}=130\right)$ within $50 \mathrm{~ns}$, using a harmonic potential along with a force constant of $5 \mathrm{~kJ} / \mathrm{mol}$. The membrane-spanning cylinder was defined with $R_{\text {cyl }}=0.6 \mathrm{~nm}$ and $Z_{\text {cyl }}=3.2 \mathrm{~nm}$. The width of the switching region in the radial direction was taken as $w=0.2 \mathrm{~nm}$, and the respective width along $z$ was taken as $h=0.6 \mathrm{~nm}$. Thirty umbrella windows were evenly distributed along the reaction coordinate, and umbrella potentials with a force constant of $5 \mathrm{~kJ} / \mathrm{mol}$ were applied. Each umbrella simulation was conducted for 300 ns, and PMFs were calculated using the g_wham method ${ }^{32}$ from the last $25 \mathrm{~ns}$ of the trajectories. For system UmbrellaM8, PMFs were calculated from the $30 \mathrm{~ns}$ trajectory, since the membrane disintegrated in several umbrella windows after this time.

Slice Coordinate. A second set of umbrella sampling simulations was performed using a recently proposed reaction coordinate, $\xi$, where $\xi$ is defined using a membrane-spanning cylinder that is decomposed into $N_{s}$ slices taken symmetrically around the center of mass of the hydrophobic membrane atoms along the membrane normal. Effectively, $\xi$ defines the fraction of slices that are occupied by polar atoms, and can take values from 0 up to 1 :

$$
\xi=N_{s}^{-1} \sum_{s=0}^{N_{s-1}} \delta_{s}\left(N_{s}^{(\mathrm{p})}\right)
$$

where $N_{s}^{(\mathrm{p})}$ denotes the number of polar heavy atoms in slice $s$, and $\delta_{s}$ is a continuous indicator function $\left(0 \leq \delta_{S}<1\right)$ that equals zero if $N_{s}^{(\mathrm{p})}=0$ and has a value close to unity if $N_{s}^{(\mathrm{p})} \geq 1$. Technical details about $\xi$ and its performance have been discussed previously. ${ }^{33}$ This method has been shown to be efficient for PMF calculations for pore formation in membranes.

PMFs were computed along $\xi$, using the technique of umbrella sampling. Starting frames for umbrella sampling were taken from a "slow-growth" pore-opening simulation, where the pore was opened by pulling the system along $\xi$ with a harmonic potential (force constant $=2000 \mathrm{~kJ} \mathrm{~mol}^{-1}$ ). The minimum of the harmonic potential was moved with a constant velocity from $\xi=$ 0 at time 0 to $\xi=1$ at $45 \mathrm{~ns}$. Umbrella sampling was performed using 24 umbrella windows. A tighter spacing of higher umbrella force constants was used for $\xi \geq 0.7$, compared to $\xi<0.7$. Accordingly, 11 windows were distributed between $\xi=0$ and $\xi=$ 0.65 in steps of 0.065 , using a force constant of $5000 \mathrm{~kJ} \mathrm{~mol}^{-1}$, and additional 13 windows were distributed between $\xi=0.7$ and $\xi=1$ in steps of 0.025 , using a force constant of $10000 \mathrm{~kJ} \mathrm{~mol}^{-1}$. Each umbrella window was simulated for $50 \mathrm{~ns}$. Following the notation described previously, ${ }^{33}$ we used $R_{\mathrm{cyl}}=1.2 \mathrm{~nm}, N_{s}=30$, $d_{s}=0.1 \mathrm{~nm}$, and $\zeta=0.75$.

Force Field Parameters and Simulations Details. All the force field parameters were taken from our previous publication. ${ }^{16}$ In short, we used the Berger force field parameters $^{34,35}$ for POPC and PATA-C12. Particularly, the quaternary ammonium group was described with the same parameters as those used for the $-\left(\mathrm{CH}_{2}\right) \mathrm{N}^{+}\left(\mathrm{CH}_{3}\right)_{3}$ moiety of the choline group in POPC. The atomic charges were recalculated using the $\mathrm{HF} / 6-31 \mathrm{G}^{*}$ level of theory, which is compatible with the Berger force field. Atom names, types, and associated partial charges are presented in Figure S2 and Table S1. The simple point charge (SPC) water model was used, ${ }^{36}$ and OPLS parameters were used for the $\mathrm{Cl}^{-}$ions. ${ }^{37}$

All simulations were performed with the GROMACS 5.1 simulation software. ${ }^{38,39}$ The SETTLE algorithm ${ }^{40}$ was used to constrain water bonds and angles. All other bonds were constrained using LINCS. ${ }^{41}$ All simulations were performed at $310 \mathrm{~K}$, using a stochastic dynamics integrator, with the exception of simulations performed for the diffusion constant estimation, for which the leapfrog integrator, together with the NoséHoover ${ }^{42,43}$ thermostat, was used. The pressure was controlled at 1 bar using a semi-isotropic Parrinello-Rahman barostat ${ }^{44}(\tau$ $=1 \mathrm{ps}$ ). Dispersive interactions and short-range repulsion were described by a Lennard-Jones potential with a cutoff of $1.4 \mathrm{~nm}$. Electrostatic interactions were calculated by applying the particle-mesh Ewald (PME) method, ${ }^{45}$ using a $1.4 \mathrm{~nm}$ realspace cutoff and $0.12 \mathrm{~nm}$ Fourier grid. 


\section{RESULTS}

MD Simulations. Long Equilibrium Simulations. Long equilibrium MD simulations were performed to study the entry of the polycation into the lipid membrane and to understand the influence of the density of the PATA-C12 repeating units per POPC molecules $\left(d_{\text {unit/POPC }}\right)$ on the properties of the POPC bilayer. In all systems, the PATA-C12 molecules were initially placed close to the membrane/water interface. We observe that the PATA-C12 dodecyl chains penetrated into the membrane hydrophobic core within a few nanoseconds of the simulations. For systems M1-M6, the PATA-C12 molecules remained in tight contact with the bilayer surface for the rest of the simulation time and were surrounded by the $\mathrm{Cl}^{-}$counterions in the aqueous phase, as shown in Figure 2 for system M1.

To monitor the insertion of PATA-C12 into the POPC membrane, mass density profiles for the nitrogen $(\mathrm{N})$ atoms and the terminal $\mathrm{CH}_{3}$ groups of dodecyl chains of the polymer (CCL), as well as for POPC double bonds $(\mathrm{C}=\mathrm{C})$ and phosphate groups (P8), were calculated (Figure S3 in the Supporting Information). For M1-M6 systems, the profiles of $\mathrm{N}$ atoms strongly overlap with the $\mathrm{P} 8$ profiles, indicating that the main chains of the polycations occupy the region around the lipid headgroups. Profiles of CCL overlap with the $\mathrm{C}=\mathrm{C}$ profiles as the alkyl moieties of the dodecyl polycation chains are immersed in the membrane reaching the double bonds in the oleyl tails. We note that, with the increase in PATA-C12 concentration, the maxima at $\sim 4 \mathrm{~nm}$ for CCL and $\mathrm{N}$ profiles increase, indicating the tendency of PATA-C12 side chains to remain partially in the aqueous phase at higher concentrations.

In the case of M8, we observe spontaneous formation of pores in the bilayer at an early stage of simulation (Figure S4 in the Supporting Information). Consequently, the distributions of $\mathrm{N}$ atoms (Figure 3, green curve) and CCL (Figure 3, black curve) for M8 are much broader, compared to those of systems with lower N (Figure S3 in the Supporting Information; the green/ black curves represent data for M1-M6), and have several maxima, indicating the presence of the polycation in both the hydrophobic membrane core and the water phase. In addition,

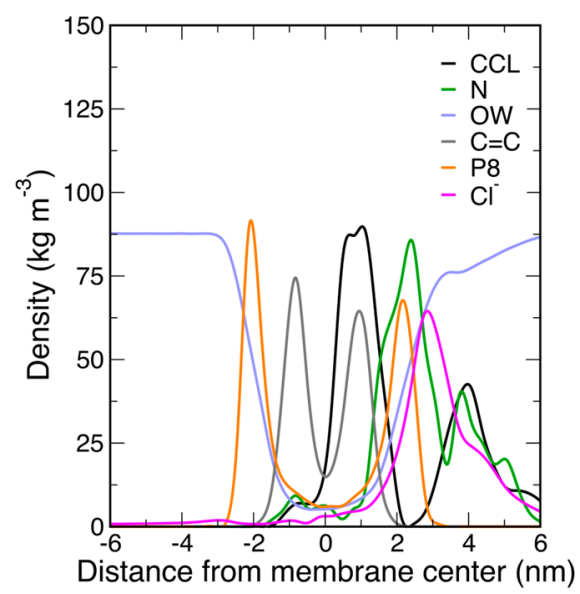

Figure 3. Scaled mass density profiles for selected groups of atoms in the M8 polycation-membrane system along the bilayer normal averaged over the entire trajectory: $\mathrm{N}$ atoms and the terminal $\mathrm{CH}_{3}$ groups of dodecyl chains (CCL) for the PATA-C12 molecules, chloride anions $\left(\mathrm{Cl}^{-}\right)$, double bonds of the oleyl chain $(\mathrm{C}=\mathrm{C})$ and phosphate groups (P8) of the POPC molecules, and water (OW) molecules. Densities for OW were scaled by 0.1 and those of CCL and $\mathrm{N}$ were scaled by 10 . the mass density profiles of the lipid phosphate groups (orange curve) and water molecules (cyan curve) demonstrate that both moieties penetrate into the hydrophobic region of the membrane, confirming the formation of transmembrane pores.

Next, we considered the effect of $d_{\text {unit/POPC }}$ on the lipid organization in the POPC bilayer. Figures $4 \mathrm{~A}$ and $4 \mathrm{~B}$ show APL and membrane thickness, respectively, as a function of $N$. We find that the thickness of the POPC bilayer decreases, while the corresponding APL strongly increases with increasing N. Since the polycations were embedded in the outer leaflet, it was instructive to compare the APL values for each leaflet in all systems (Figure S5 in the Supporting Information). We find that APLs for the inner and outer leaflets differ only for large N (M4 and M6), indicating that the addition of more polycation molecules slightly increased the asymmetry in the APL between the outer and inner leaflets. Although the area asymmetry between the two leaflets is negligible $(<0.7 \%$ for $N=6)$, it is consistent with previous observations. The asymmetric insertion of molecules into lipid vesicles involves an area expansion of the outer monolayer, with respect to the inner monolayer, which is often accompanied by positive-curvature strain, depending on the shape of the inclusion and its packing efficiency with the background lipids. ${ }^{46}$

To quantify the structural orientation and flexibility of the lipid acyl chains, we calculated the deuterium order parameters $\left(\left|S_{\mathrm{CD}}\right|\right)$ of the palmitoyl groups of the POPC molecules in the outer leaflet (Figure 4C). In all systems, the addition of PATAC12 led to disorder in the part of lipid tails located close to the headgroup (carbon atoms $\mathrm{C} 1-\mathrm{C} 5$ ), and the disorder in this part is concentration-dependent. At the lowest concentration of PATA-C12 $(N=1)$, the $\mathrm{C} 6-\mathrm{C} 13$ part of the lipid acyl chain is only slightly disordered (Figure 4C, blue curve), compared to the pure POPC membrane. However, increasing $N$ has a stronger effect on the $\mathrm{C} 6-\mathrm{C} 13$ part, as observed for $N=2,4$, and 6 (Figure 4C; purple, red and green curves, respectively). The deuterium order parameters calculated for both leaflets are shown in Figure S6 in the Supporting Information.

The mobility of PATA-C12 molecules adsorbed on the membrane surface can be quantified by their lateral diffusion constants. Hence, we calculated these quantities for the polycation $\left(D_{\text {PATA-C12 }}\right)$ and the lipids $\left(D_{\text {POPC }}\right)$ for the M1M6 systems (Figure $S 1$ and Table 2 ). For $N=1, D_{\text {PATA-C12 }}$ is comparable to $D_{\text {POPC }}$. However, an increase in $N$ results in 2 -fold reduction of $D_{\text {PATA-C12 }}$, indicating slower diffusion of polycation molecules with increasing $d_{\text {unit/POPC }}$. Note that all polycation molecules remain strongly inserted in the membrane bilayer through the simulations. The lateral movement of PATA-C12 molecules is hindered due to crowding effects and strong repulsive interactions between highly charged polymer molecules. Interestingly, $D_{\mathrm{POPC}}$ for systems $\mathbf{M 1 - M 6}$ is similar to that for the pure POPC membrane, suggesting that the adsorption of PATA-C12 molecules on the membrane has very little effect on the lateral diffusion of lipids.

The asymmetric adsorption of the polycation molecules causes an accumulation of strong positive charges on a single membrane leaflet. Ideally, the $\mathrm{Cl}^{-}$counterions present in the aqueous phase would compensate these positive charges. To elucidate the mutual position of the polymer quaternary ammonium groups and $\mathrm{Cl}^{-}$ions (hence, the degree of charge compensation), we calculated the mass density profiles of the $\mathrm{N}$ atoms of the PATA-C12 molecules and $\mathrm{Cl}^{-}$ions across the membranes (green and magenta curves in Figure 3 and Figure $\mathrm{S} 3$ ). The $\mathrm{Cl}^{-}$profiles have maxima at a distance of $\sim 2.8 \mathrm{~nm}$ from 

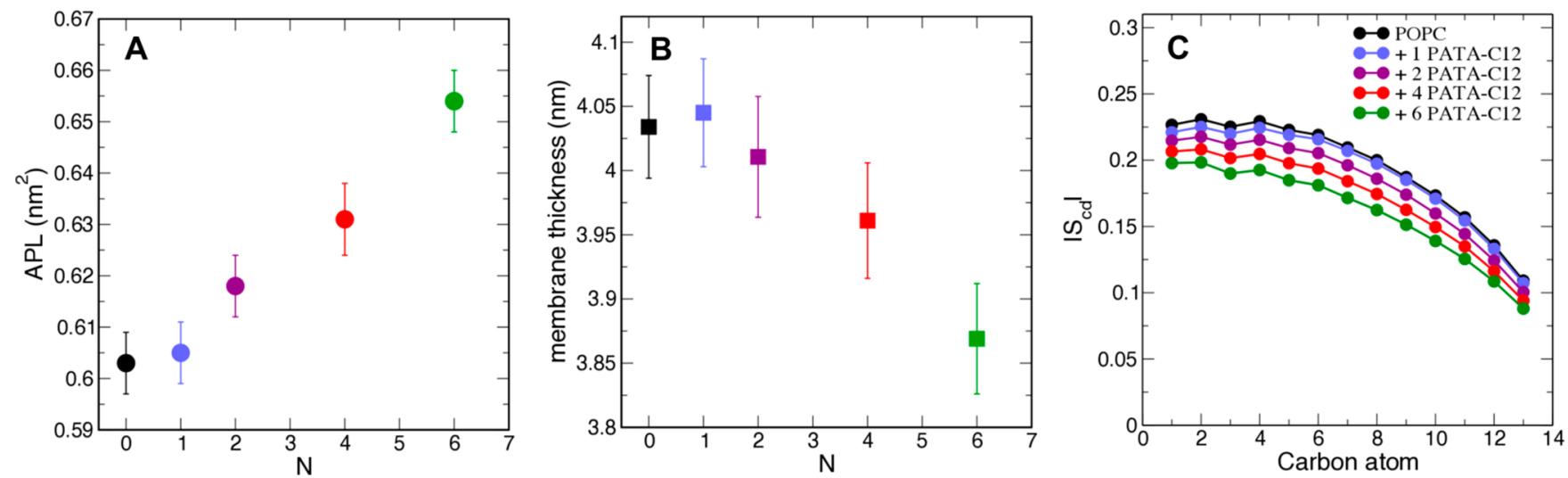

Figure 4. Membrane properties, as a function of the number of PATA-C12 molecules in the system, N: (A) area per lipid (APL), (B) membrane thickness, and $(\mathrm{C})$ deuterium order parameter $\left(\left|S_{\mathrm{CD}}\right|\right)$ for the POPC palmitoyl chains in the outer leaflet. For $N=8$, the membrane was ruptured within a few nanoseconds. The $I S_{\mathrm{CD}} \mid$ values are the average calculated for all lipids in the bilayer.

Table 2. Lateral Diffusion Constants $(D)$ of POPC Lipids and PATA-C12 Molecules ${ }^{a}$ Adsorbed on the Bilayer ${ }^{b}$

$\begin{array}{lcc}\text { system } & D_{\text {PATA-C12 }}\left[\times 10^{7} \mathrm{~cm}^{2} / \mathrm{s}\right] & D_{\text {POPC }}\left[\times 10^{7} \mathrm{~cm}^{2} / \mathrm{s}\right] \\ \text { POPC } & - & 0.26 \pm 0.01 \\ \text { M1 } & 0.30 \pm 0.01 & 0.31 \pm 0.05 \\ \text { M2 } & 0.13 \pm 0.01 & 0.24 \pm 0.01 \\ \text { M4 } & 0.14 \pm 0.03 & 0.28 \pm 0.02 \\ \text { M6 } & 0.14 \pm 0.04 & 0.32 \pm 0.10\end{array}$

${ }^{a_{T}}$ The mean \pm standard error from three independent simulations is shown. ${ }^{b}$ Here, M1, M2, M4, and M6 correspond to the systems with $N=1,2,4$, and 6 PATA-C12 molecules, respectively.

the bilayer center and are partly overlapped by the profiles of the $-\mathrm{N}\left(\mathrm{CH}_{3}\right)_{3}{ }^{+}$groups of the polycation (green curves in Figure 3). Furthermore, comparing with the P8 atoms of the lipid headgroups (orange curves in Figure 3 and Figure S3), we observed that the $\mathrm{Cl}^{-}$anions are preferentially located outside the membrane. Taken together, this indicates that the positively charged groups of PATA-C12 that are located deeper in the polar region of the bilayer are not entirely compensated by the counterions and, hence, can give rise to an electrostatic field between the membrane leaflets. Therefore, we calculated the electrostatic potential across the membrane for the M1-M6 systems, as shown in Figure 5A. The profile for the pure POPC system (black curve in Figure 5A) reveals the existence of a small positive potential difference between the membrane core and the adjacent water phases, in agreement with experiments and previous MD simulations. ${ }^{47}$ We calculated the voltage between the membrane leaflets (referred to as "interleaflet voltage") as a potential difference between the two leaflets, which are represented as distinct maxima on the potential curves. As may be expected, $d_{\text {unit/POPC }}$ causes an increase in the positive potential at the outer leaflet, and, thus, an increase in the interleaflet voltage, ranging from ca. $190 \mathrm{mV}$ for the M1 system to $360 \mathrm{mV}$ for the system with six PATA-C12 molecules. The interleaflet voltage for the M8 system (Figure 5B), calculated from the initial part of the trajectory (corresponding to the intact membrane), is $\sim 600 \mathrm{mV}$. Here, note that the interleaflet voltage reported here differs from the transmembrane voltage recorded in experiments and simulations of membranes under an applied electric field. ${ }^{47}$ In the latter, there is an electrostatic potential difference between the two sides (bulk regions) of a membrane. In our case, the transmembrane voltage is equal to $0 \mathrm{~V}$, as evident in Figure 5, although the asymmetric distribution of charges
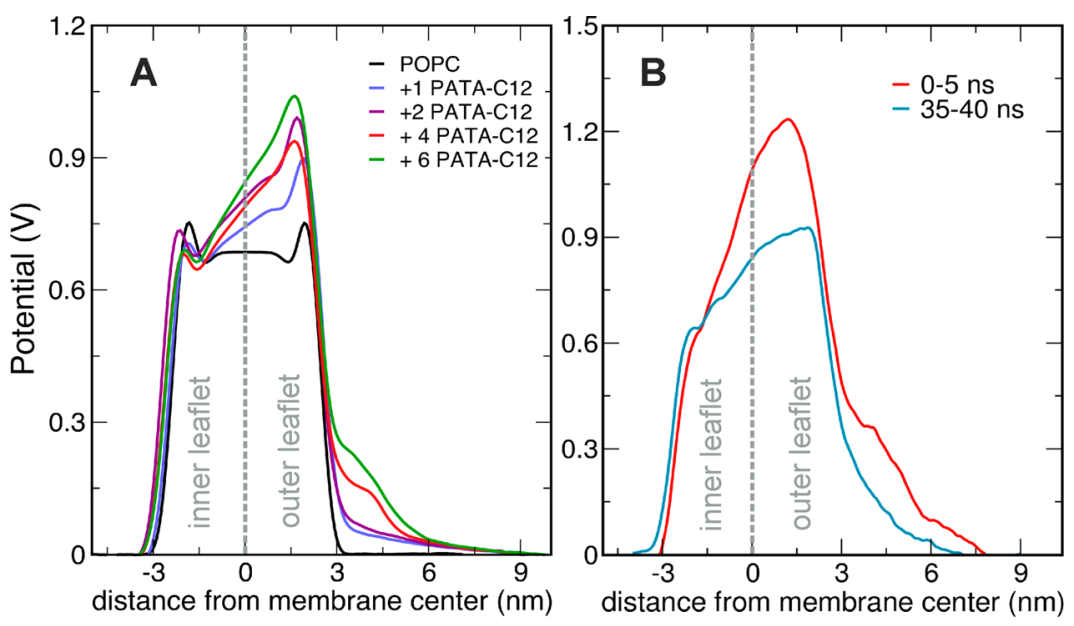

Figure 5. Electrostatic potential across the POPC membrane in the presence of various concentrations of PATA-C12 molecules, N: (A) for the M1M6 systems, (B) for the M8 system calculated at the initial (no spontaneous pores) and final (with spontaneous pores) parts of the trajectory. The potential across the POPC membrane in the absence of PATA-C12 is shown in black as a reference. PATA-C12 molecules were adsorbed on the outer leaflets of the POPC membrane. Note the different $y$-axis scale in panel (B). 


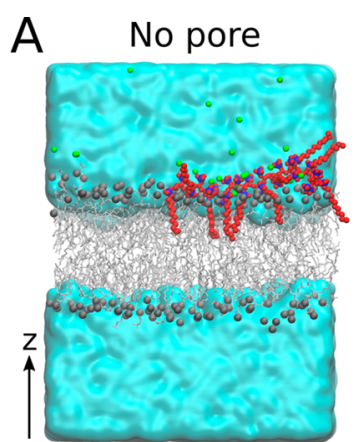

B

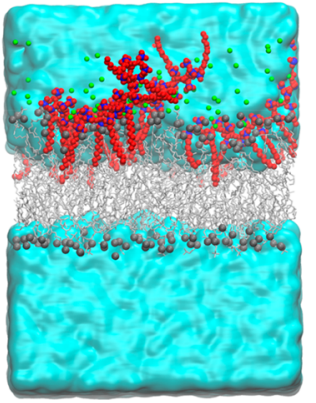

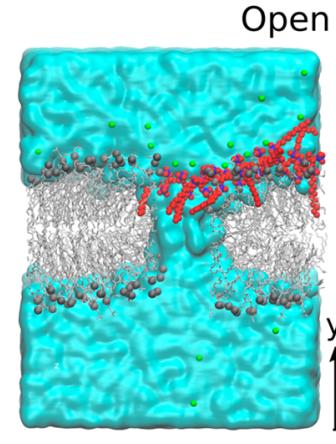
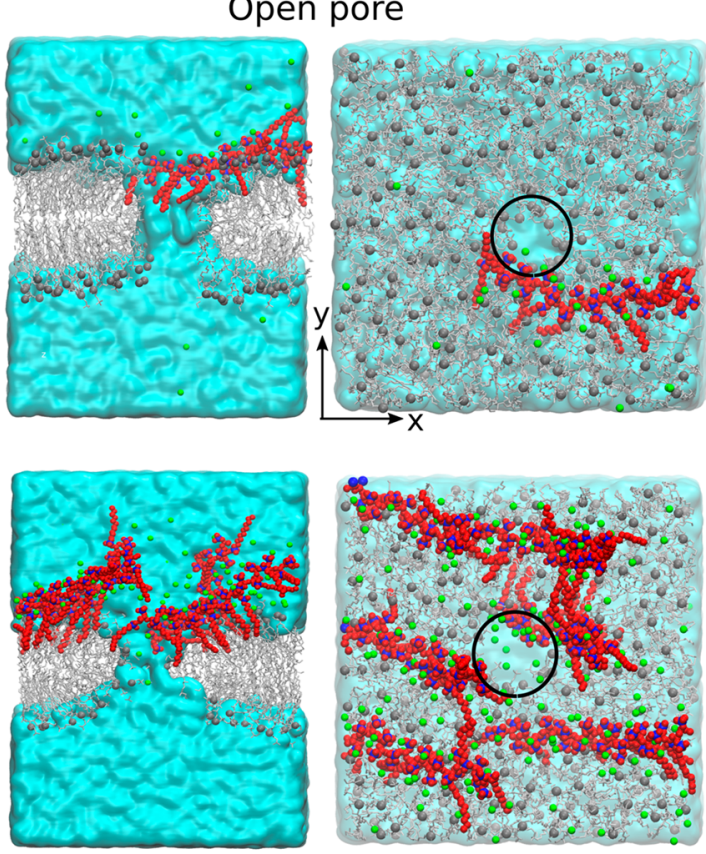

Figure 6. Snapshots from umbrella sampling simulations for systems UmbrellaM1 and UmbrellaM6. Equilibrium configuration ("no pore") and configuration with "open pore" in the membrane corresponding to the final umbrella window (side and top views) for systems (A) UmbrellaM1 and (B) UmbrellaM6. Open pores in the $x y$ planes are marked with black circles, pores are toroidal in shape, and lined by lipid headgroups. POPC molecules are shown as gray sticks with phosphate groups shown as gray spheres. PATA-C12 molecules are shown as red spheres with nitrogen atoms in blue. Green spheres represent $\mathrm{Cl}^{-}$ions, and cyan surface represent water.

induces a potential difference between the leaflets that we termed as the interleaflet voltage.

To summarize, we found that the increase in density of PATAC12 units on the POPC membrane induces changes in membrane properties such as diminished thickness and reduced lipid packing, slower polycation diffusion, and increasing disorder in the POPC acyl chains. In addition, the asymmetric incorporation of the polycation to the outer membrane leaflet generates the electrostatic potential gradient within the membrane and its value increases with increasing $d_{\text {unit/POPC. At }}$

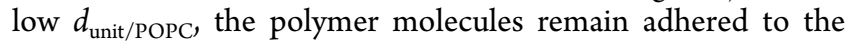
bilayer over several hundred nanoseconds and we did not observe any spontaneous pore formation in the membrane. For the largest membrane coverage by the polycation units $\left(d_{\text {unit/POPC }} \approx 0.8\right.$, system $\left.\mathbf{M 8}\right)$, spontaneous pore nucleation is observed at a very early stage (after ca. $5 \mathrm{~ns}$ ) and multiple transmembrane pores nucleated within $30-40 \mathrm{~ns}$. The polymer chains penetrated partially around such pores, thus forming a hydrophilic pore lining, as shown in Figure S4.

Umbrella Sampling Simulations. To evaluate the role of PATA-C12 in the formation of transmembrane pores, PMFs for pore formation were calculated for the pure POPC bilayer and POPC bilayers with different $N$ (systems UmbrellaM1UmbrellaM8). Initial configurations used in the calculations are shown in Figure S7 in the Supporting Information. Figure 6 shows representative snapshots taken from the umbrella sampling simulations for initial (denoted as "no pore") and final (corresponding to the final umbrella windows, denoted as "open pore") configurations of the systems UmbrellaM1 (Figure 6A) and UmbrellaM6 (Figure 6B). We observed that the PATA-C12 molecules remained inserted near the pore and did not translocate to the inner leaflet.

PMFs for pore formation as a function of the number of water molecules in the membrane-spanning cylinder, $N_{\mathrm{w}}$ (see the
Methods section), are shown in Figure 7. All PMFs show three distinct regions. At small $N_{w}$, the PMFs are quadratic functions

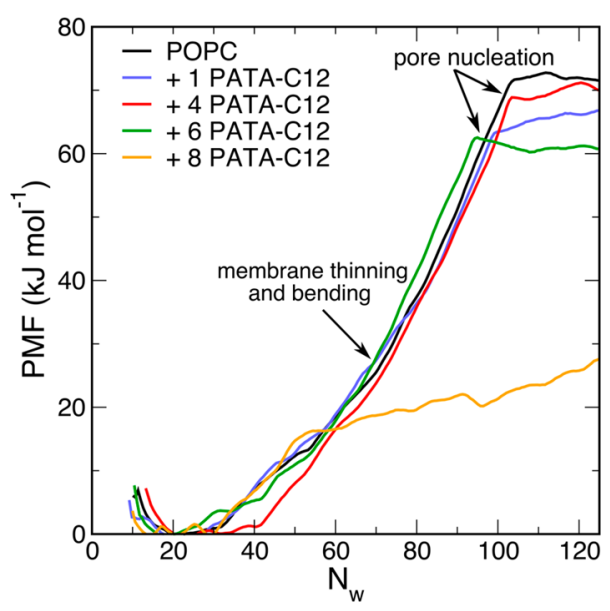

Figure 7. PMFs of pore nucleation in POPC membranes containing different concentrations of PATA-C12 using the water-density coordinate. PMFs were computed from the last $25 \mathrm{~ns}$ of the $300 \mathrm{~ns}$ trajectory for each umbrella windows. For system M8, PMFs were calculated from the $30 \mathrm{~ns}$ trajectory. The addition of $N=1$ and 6 PATA$\mathrm{C} 12$ chains lowered the free energy for pore formation $\left(\Delta G_{\text {pore }}\right)$. For $N$ $=8$ the membrane pores are formed spontaneously, in line with a small $\Delta G_{\text {pore }}$ (orange curve).

of $N_{\mathrm{w}}$. This quadratic regime corresponds to the nucleation of the transmembrane pore, i.e. formation of a water defect in the membrane, and membrane deformation, i.e. thinning of the membrane and rearrangement of the lipids to form the edge surrounding the pore. This quadratic regime also denotes that pore nucleation process is accompanied by a rapid increase in 
the free energy. The kinks in the PMFs reflect the formation of a continuous water cylinder, i.e. opening of a full transmembrane pore, and they correspond to the free energy of pore formation $\left(\Delta G_{\text {pore }}\right)$. Following pore nucleation, the pore grows in size with increasing $N_{\mathrm{w}}$, as denoted by plateaus in the PMFs.

We found that $\Delta G_{\text {pore }}$ for the pure POPC membrane is $\sim 70 \mathrm{~kJ}$ $\mathrm{mol}^{-1}$, which is in reasonable agreement with the previously reported value of $78 \mathrm{~kJ} \mathrm{~mol}^{-1}$ calculated for the pure DPPC bilayer. ${ }^{26}$ In addition, a full transmembrane pore is formed when $N_{\mathrm{w}}$ exceeds 100. Our simulations reveal that PATA-C12 plays an active role in the formation of pores in the lipid membrane. The addition of polymer molecules consistently decreases the free energy of pore formation (blue curve for $N=1$ and green curve for $N=6$; see Figure 7), as compared to the pure POPC bilayer (black curve). As $N$ increases, the kinks in the PMFs show distinct shifts toward lower $N_{\mathrm{w}}$. In other words, fewer water molecules are sufficient to nucleate a transmembrane pore, indicating a decrease in membrane thickness with increasing $N$. This observation is consistent with the change in thickness observed in equilibrium simulations (Figure 4B) upon increasing $d_{\text {unit/POPC }}$. For system UmbrellaM8, stable pores spontaneously formed within $30 \mathrm{~ns}$ of the simulations. The PMF for this system reveals that the pores were formed at $\sim 50$ water molecules in the membrane-spanning cylinder, and the energy cost is $\sim 25 \mathrm{~kJ} \mathrm{~mol}^{-1}$. This is an unexpected result, since we observed spontaneous rupture of membrane for the M8 system, and hence, the PMF for $N=8$ should be much lower than $\sim 25 \mathrm{~kJ}$ $\mathrm{mol}^{-1}$. The water-density reaction coordinate keeps the defined membrane-spanning cylinder fixed, thus the reaction coordinate cannot capture the defects and pores formed outside this fixed cylinder.

Figure 8 shows the PMFs calculated using the slice coordinate. These PMFs reveal three regimes: the region around $\xi \approx 0.3$ (minimum of the PMF) corresponds to a flat bilayer, the region around $\xi \sim 0.8$ corresponds to the nucleation barrier for pore opening, and finally $\xi \approx 1.0$ corresponds to an open pore. We observe that (a) PMFs for pure POPC bilayer

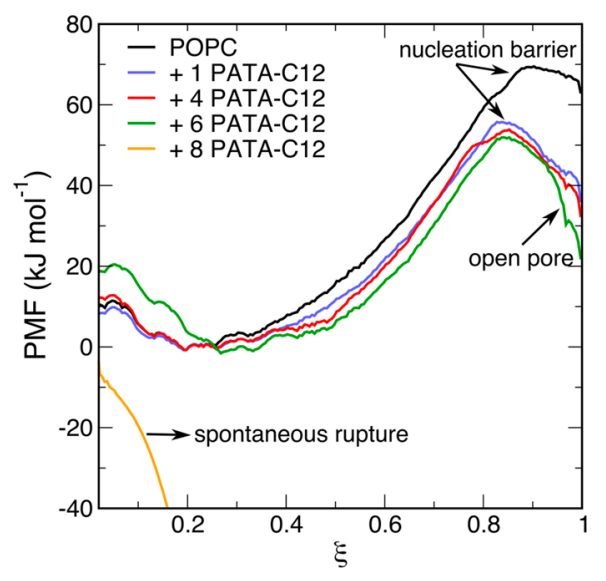

Figure 8. PMFs for pore nucleation in POPC membranes containing different numbers of PATA-C12 molecules using the slice coordinate. PMFs were computed from the entire 50 ns trajectory for each umbrella window. PMFs for POPC (black curve), and $N=1$ (blue), 4 (red), and 6 (green) PATA-C12 molecules show nucleation barriers for pore formation. The addition of increasing number of PATA-C12 molecules, $\mathrm{N}$, to the POPC membrane lowers the free energy for pore formation $\left(\Delta G_{\text {pore }}\right)$. For $N=8$, there is spontaneous rupture of the membrane in agreement with equilibrium simulations, and rationalized by a negative $\Delta G_{\text {pore }}$ (orange curve).
(Figure 8, black curve), and for bilayers with $N=1,4$, and 6 (blue, red, and green curve, respectively), PATA-C12 molecules show nucleation barriers for pore formation, and (b) the addition of increasing $N$ reduces the free energy for pore formation $\left(\Delta G_{\text {pore }}\right)$. Nucleation barriers and $\Delta G_{\text {pore }}$ are summarized in Table 3. Furthermore, $\Delta G_{\text {pore }}$ for the pure

Table 3. Summary of Pore Opening Thermodynamics with Increasing Concentration of PATA-C12, Using the Slice Coordinate $^{a}$

\begin{tabular}{ccccc} 
system & $\begin{array}{c}\text { density of } \\
\text { the } \\
\text { PATA-C12 } \\
\text { molecules, } N\end{array}$ & $\begin{array}{c}\text { polycation, } \\
d_{\text {unit/POPC }}\end{array}$ & $\begin{array}{c}\text { pore nucleation } \\
\text { free energy, } \\
\Delta G_{\text {pore }} \\
{\left[\mathrm{kJ} \mathrm{mol}^{-1}\right]}\end{array}$ & $\begin{array}{c}\text { nucleation } \\
\text { barrier } \\
{\left[\mathrm{kJ} \mathrm{mol}^{-1}\right]}\end{array}$ \\
\hline Pure POPC & 0 & 0 & 65 & 70 \\
UmbrellaM1 & 1 & 0.10 & 38 & 56 \\
UmbrellaM4 & 4 & 0.42 & 32 & 53 \\
UmbrellaM6 & 6 & 0.62 & 22 & 51 \\
UmbrellaM8 & 8 & 0.83 & $<0$ & -
\end{tabular}

${ }^{a}$ Pore nucleation free energies, $\Delta G_{\text {pore }}$ and nucleation barriers for different number of PATA-C12 molecules $(N)$. Data refers to the PMFs shown in Figure 8.

POPC bilayer is $\sim 65 \mathrm{~kJ} \mathrm{~mol}^{-1}$ (black curve in Figure 8 ), in agreement with the value calculated from the water-density coordinate (see black curve in Figure 7 ). With increasing $N$ $\left(d_{\text {unit/POPC }}\right), \Delta G_{\text {pore }}$ strongly decreases, eventually leading to spontaneous rupture of the bilayer for $N=8$ (Figure 8, orange curve). In fact, for $d_{\text {unit/POPC }} \approx 0.6, \Delta G_{\text {pore }}$ decreases by almost $40 \mathrm{~kJ} \mathrm{~mol}^{-1}$ (green curve), compared to the pure POPC bilayer. We also notice that the strongest change in $\Delta G_{\text {pore }}$, with respect to the pure POPC bilayer is observed for $N=1$ (Table 3, second column). In addition, the nucleation barrier decreases with $d_{\text {unit/POPC }}$ (blue, green, and red curves, with respect to the black curve), indicating that the addition of PATA-C12 increases the propensity to nucleate pores. Furthermore, if the umbrella potentials are removed, an open pore in the POPC membrane (black curve) would close within a shorter time scale, compared to open pores formed in the presence of PATA-C12. In other words, pores formed in the presence of PATA-C12 would have longer lifetimes and are likely to be more stable than those formed in the pure POPC bilayer.

Taken together, our PMFs indicate that $\Delta G_{\text {pore }}$ decreases with increasing $d_{\text {unit/POPC}}$, and a sufficiently high density $\left(d_{\text {unit/POPC }} \sim\right.$ 0.8 ) of the polycation adsorbed on the membrane can lead to several pores or even to membrane/liposome disintegration. PATA-C12 also decreases the nucleation barrier to open stable pores.

PATA-C12 - POPC Monolayer Interaction. The Langmuir monolayer technique is an effective way to study the impact of polymers on lipid membranes. ${ }^{48,49}$ Although the lipid monolayer on the polymeric solution mimics only one of the membrane leaflets, it is a good model of the outer lipid layer of the liposomes exposed to the polymer. Surface pressuremean molecular area $(\pi-A)$ isotherms are measured during monolayer compression. The shape and location of these isotherms reflect the monolayer state (compression modulus) and the organization of lipid molecules (mean molecular area) in the monomolecular film.

The $\pi-A$ isotherms recorded during compression of the POPC monolayer on $1 \mathrm{mM} \mathrm{NaCl}$ and aqueous solutions of PATA-C12 at various concentrations are shown in Figure 9. On 

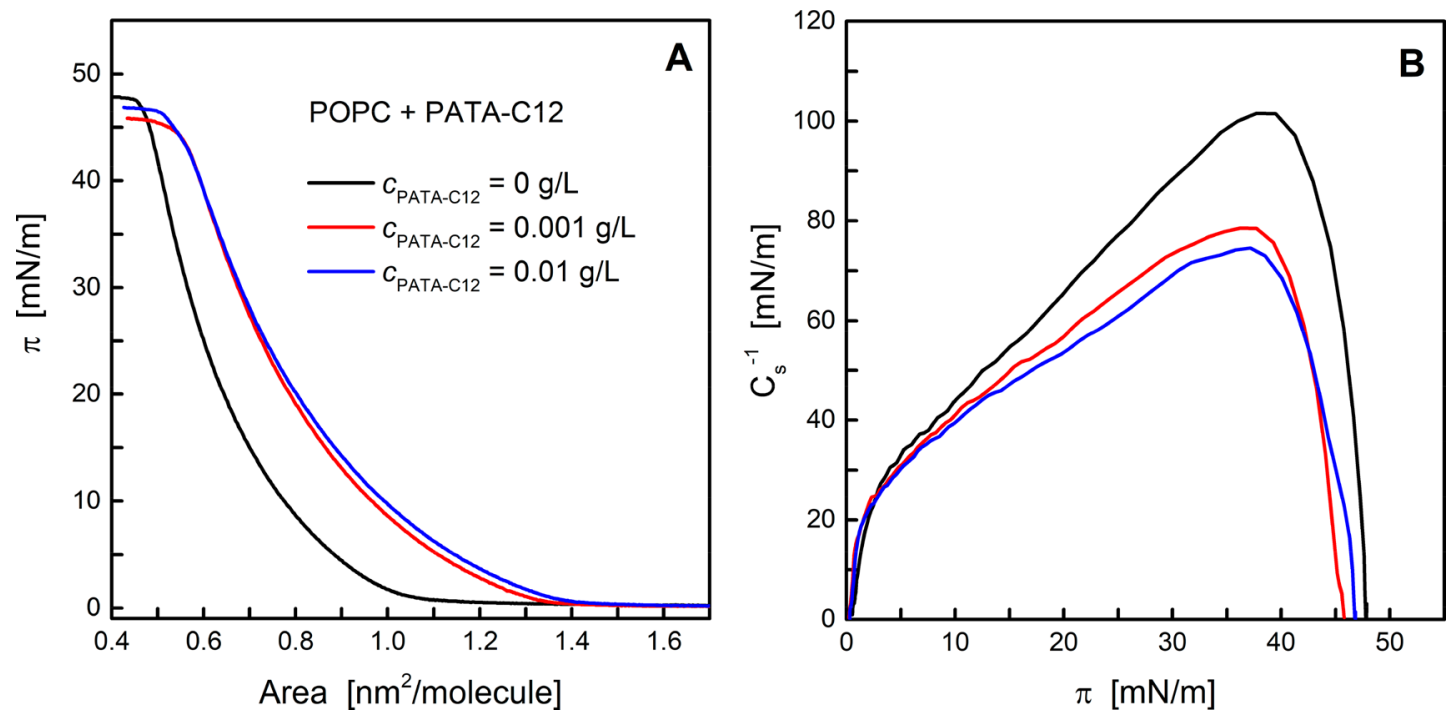

Figure 9. (A) Surface pressure $(\pi)$-area (A) isotherms recorded at $25^{\circ} \mathrm{C}$ for the POPC monolayer deposited on the $1 \mathrm{mM} \mathrm{NaCl}$ subphase of pH 7.4 containing different concentrations of PATA-C12. (B) Compression modulus $\left(C_{\mathrm{s}}^{-1}\right)$ versus surface pressure.

the basis of these isotherms, the compression modulus $\left(C_{\mathrm{s}}^{-1}\right)$ was calculated using eq 2 .

Figure 9A shows that the presence of the polycation in the subphase affects the position of the POPC isotherms. The isotherms shifted to larger molecular areas with increasing concentrations of PATA-C12, indicating that the polycations can interact with the zwitterionic membrane and change its properties. PATA-C12 incorporates into the lipid monolayer and thus increases the average area occupied by the POPC molecules. In addition, the polymer incorporation perturbs the monolayer structure, leading to increased membrane fluidity, denoted by a considerable reduction in the maximum value of $\mathrm{C}_{\mathrm{s}}^{-1}$, as shown in Figure 9B.

Interestingly, the large isotherm shift was observed already at the lower concentration of PATA-C12, and a further 10-fold increase in the polymer concentration to $0.01 \mathrm{~g} / \mathrm{L}$ had little effect on the POPC monolayer (Figure 9A). That observation could be explained by assuming PATA-C12 aggregation in an aqueous environment at higher polymer concentrations. It is well-known that hydrophobically modified polymers selfassemble into micelles in aqueous dispersions. ${ }^{50,51}$ To verify this assumption, we determined the value of critical micellar concentration (CMC) for PATA-C12 (see the Supporting Information). It was found that thermodynamically stable micelles are formed at $c_{\text {PATA-C12 }}>0.027 \mathrm{~g} / \mathrm{L}$ (Figure S9 in the Supporting Information). Thus, a possible explanation of the lower affinity of the polycation for the POPC membrane with the increasing concentration of PATA-C12 is rather related to the repulsion between the polymer-coated membrane and the polycation chains in the aqueous phase. Initially, PATA-C12 strongly adsorbs on the monolayer surface with the dodecyl tails immersed in the hydrophobic lipid core and the $-\mathrm{N}\left(\mathrm{CH}_{3}\right)_{3}^{+}$ groups exposed to the bulk solution, thereby increasing the surface potential of the POPC film from initially negative to strongly positive. ${ }^{16}$ Thus, a highly positively charged shell is formed at the surface of the lipid monolayer, which suppresses further polycation-membrane interactions.

Calcein-Release Studies. The membrane activity of polymers, in particular, their ability to form pores, can be quantified by fluorescence spectroscopy using calcein, which is a hydrophilic fluorescent dye. ${ }^{114}$ Calcein is entrapped inside liposomes at a concentration that causes self-quenching of the dye fluorescence. When the dye leaks out of the vesicles, it is diluted in the bulk aqueous phase and its emission turns on, so it can be monitored using fluorescence measurements. Figure 10

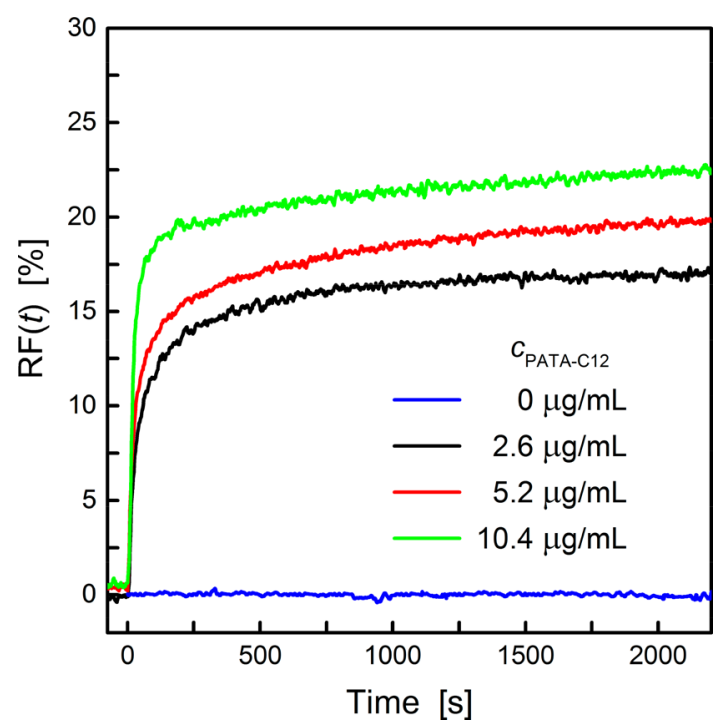

Figure 10. Time course of calcein release (RF) from the POPC liposomes $\left(c_{\mathrm{POPC}}=0.13 \mathrm{mg} / \mathrm{mL}\right)$ treated with PATA-C12 at different concentrations of $0,2.6,5.2$, and $10.4 \mu \mathrm{g} / \mathrm{mL}$ (corresponding to $d_{\text {unit/POPC }}$ values of $0,0.09,0.18$, and 0.36 ).

shows the typical time course of the RF values of calcein, calculated according to eq 1 . In the case of the pure POPC liposomes, the RF value did not change during the experiment, indicating that the permeability of the lipid bilayer for calcein is very low and that the structure of liposomes remained intact during the measurement, in agreement with previous studies, where the calcein leakage from the POPC liposomes was very slow, $\sim 1 \%$ over $3 \mathrm{~h}^{52}$ In the case of the POPC vesicles treated with PATA-C12 at various concentrations, corresponding to

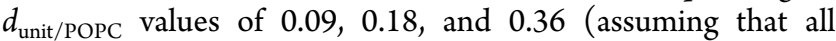
polymer is adsorbed to the liposome surface), a marked increase in the RF values was observed (Figure 10). Soon after the 


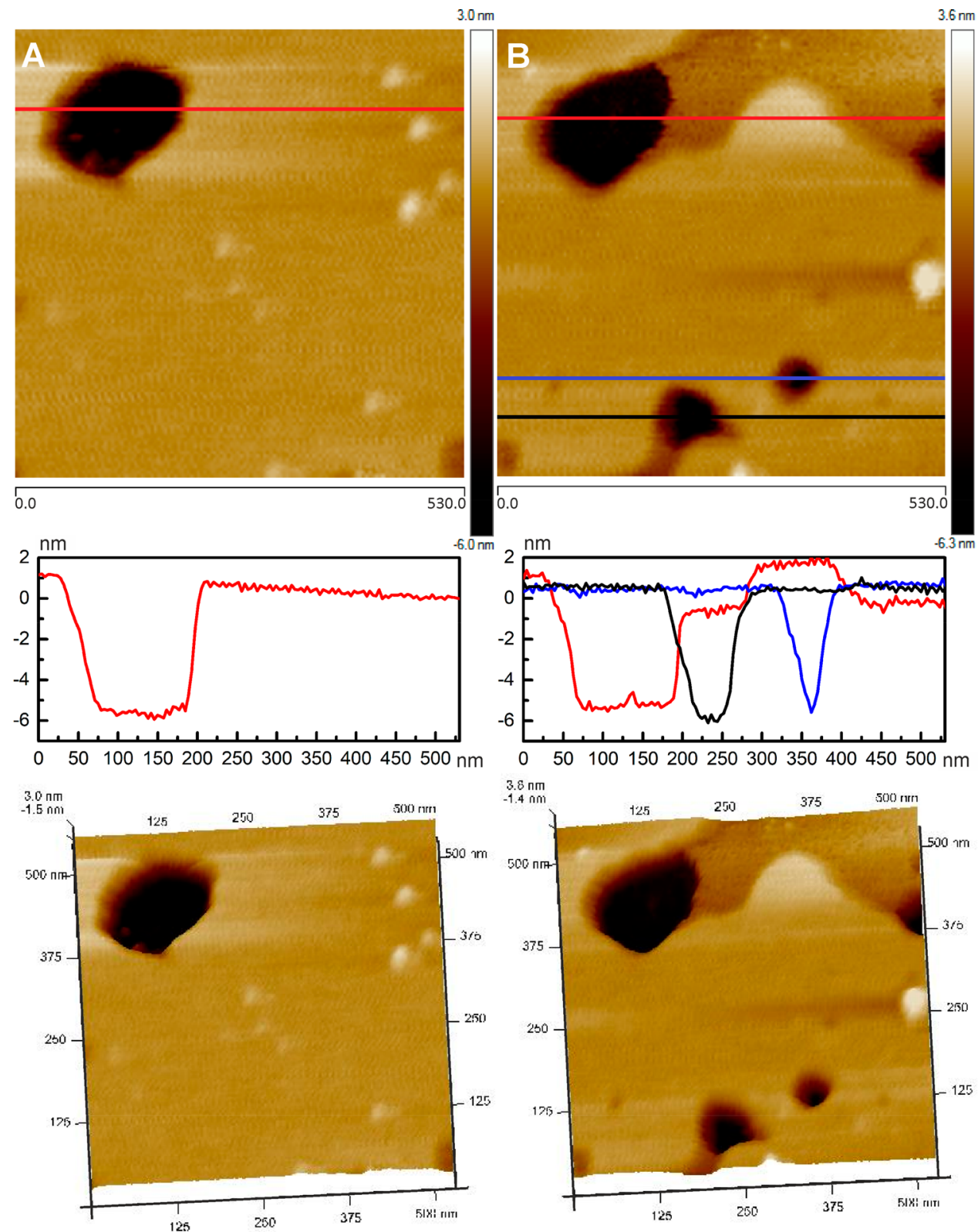

Figure 11. Representative AFM height images of the the wet state of POPC bilayers deposited on the silicon substrate (A) before and (B) after adding PATA-C12 $\left(c_{\text {PATA-C12 }} \approx 0.005 \mathrm{mg} / \mathrm{mL}\right)$. The corresponding height profiles across the bilayers (red, blue, and black lines) and 3D images are shown below.

introduction of PATA-C12, the intensity of fluorescence increased sharply and subsequently reached a constant value. However, the total leakage of calcein from the CL liposomes was only moderately dependent on the polycation concentration in the studied range. Figure 10 shows that $15 \%$ of calcein was released from the POPC liposomes at a very low PATA-C12 concentration of $0.0026 \mathrm{mg} / \mathrm{mL}$. A 4-fold increase in PATAC12 concentration to $0.0104 \mathrm{mg} / \mathrm{mL}$ caused only a 1.3 fold increase in the amount of calcein released. These results are consistent with the monolayer experiments that showed a decrease in the affinity of PATA-C12 to the zwitterionic membrane as the polycation concentration increased. The diameter of calcein was estimated to be $1.3 \mathrm{~nm},{ }^{53}$ and therefore, the size of pores induced by the polycation should be greater than this value to allow the passage of the dye molecules. These results clearly demonstrate that PATA-C12 interacts with the
POPC membrane, increasing its permeability to polar compounds in a concentration-dependent manner.

Atomic Force Microscopy (AFM) Studies. AFM microscopy can be employed to directly observe the effect of polycations on lipid membranes. AFM of supported lipid bilayers (SLBs) treated with various polymers revealed that the cationic polymer can cause the formation of nanoscale holes in previously intact bilayers or removal of lipid molecules from edges of existing bilayer defects. ${ }^{7}$ Figure 11 shows AFM height images of the supported POPC membrane before and after injection of the PATA-C12 solution. The initially formed POPC bilayer contained holes resulting from incomplete fusion of liposomes. The presence of these holes is quite useful to verify bilayer formation and its thickness. ${ }^{10}$ The depth of this defect is $\sim 6 \mathrm{~nm}$, which corresponds to the thickness of the hydrated lipid membranes (that include a thin layer of water between the bilayer and the support) observed with AFM. ${ }^{54}$ After the 
addition of PATA-C12, new small holes appeared in the previously intact area of the membrane (Figure 11B and Figure S10 in the Supporting Information). The typical hole sizes observed by AFM ranged between $30 \mathrm{~nm}$ and $60 \mathrm{~nm}$. Presumably, holes with smaller diameters were also formed, but the size of scanning tip $(\sim 20 \mathrm{~nm})$ was too large to enable observation of them. In addition to that, the bilayer thickness was reduced to $\sim 5.0 \mathrm{~nm}$ in some areas (see the red line in Figure 11B). This indicates that PATA-C12 is probably adsorbed unevenly on the membrane surface, creating patches with reduced thickness. Nonuniform adsorption of polycation (socalled "polymer patches") on zwitterionic liposomes was previously proposed to explain aggregation of polymerdecorated lipid vesicles. ${ }^{3,49}$ We did not observe the expansion of pre-existing holes, indicating that PATA-C12 did not remove lipid molecules from the edges of existing bilayer defects.

AFM observation revealed that PATA-C12 is able to reduce the membrane thickness and to porate the POPC membrane by forming nanoscale holes. As a result, the lipid membrane permeability for polar compound should considerably increase after the polycation addition.

\section{DISCUSSION}

We investigated the interaction between a strong linear polycation modified with the dodecyl side groups (PATA$\mathrm{C} 12$ ) and the zwitterionic lipid membrane, using a combination of atomistic-scale $\mathrm{MD}$ simulations, including free-energy calculations, and experimental techniques. PATA-C12 has been previously shown to adhere strongly to zwitterionic POPC membrane. ${ }^{16}$ However, there is hardly any quantitative data on membrane pore formation in the presence of hydrophobically modified polycations. Here, we characterize the molecular mechanism of the interaction of the polycation with the POPC membrane and its ability to induce membrane pores. In our study, we used very low polycation concentrations (in the range of $0.001-0.01 \mathrm{mg} / \mathrm{mL}$ ), thus much below the CMC of PATA-C12, to avoid micelle formation and disruption of the liposomes, because of the increase in osmotic pressure across the semipermeable lipid bilayer. Dynamic light scattering (DLS) and nanoparticle tracking analysis (NTA) measurements (the Supporting Information) revealed that the POPC liposomes retained their integrity at low PATA-C12 concentrations. This is consistent with our previous observations performed using direct visualization with cryo-transmission electron microscopy. ${ }^{16}$

Strong PATA-C12-POPC Membrane Interactions. It is known that the presence of hydrophobic groups in the structure of strong polycations is necessary for efficient adhesion of these polymers to the surface of zwitterionic membranes. ${ }^{14,16}$ Our equilibrium MD simulations show that the PATA-C12 molecules readily adsorb on the membrane surface, and their alkyl side chains are mostly buried in the hydrophobic core of the membrane, while the main polymer chain is located at the water/bilayer interface (see the Supporting Information for an animation from the trajectory for system M1). Despite the dynamic behavior of lipids and PATA-C12 molecules, this arrangement remained unaltered in the microsecond time scale of equilibrium MD simulations, indicating a tight binding between the PATA-C12 molecules and the POPC bilayer. This tight binding is a consequence of hydrophobic interactions between the PATA-C12 alkyl chains and the POPC lipid tails, which, in turn, place the polymer in an optimal position for electrostatic interactions between its positively charged quaternary ammonium groups and negatively charged phosphate groups of the lipids. The calculated mass density profiles additionally confirm this observation.

PATA-C12 Affects the Membrane Structure. The adsorption of PATA-C12 molecules strongly affects membrane properties (acyl chain order, thickness, and packing) in a concentration-dependent manner. As observed with AFM, the thickness of POPC membrane decreases due to PATA-C12 adsorption. In fact, for system M6, the membrane thickness decreased by $\sim 0.17 \mathrm{~nm}$, and the area per lipid increased by $8 \%$, in comparison to the respective values for a pure POPC membrane. Hence, the adhesion of PATA- $\mathrm{C} 12$ reduces the packing of the POPC membrane. This result is consistent with the experimental observation of a shift in the POPC isotherm toward higher molecular areas for the lipid monolayers compressed on the polycation-containing subphases. These experiments also show the reduction in the maximum values of the compression modulus $\left(C_{s}^{-1}\right)$ for the monolayers recorded on the polycation solutions, suggesting an increase in membrane fluidity. Increased fluidity of the membrane can be captured in MD simulations by either increased disorder of the lipid acyl chains, or by increased lateral diffusion of lipids, or both. The order parameters, averaged over the lipids in the outer leaflet, show that the presence of PATA-C12 on the membrane surface caused significant disordering of the lipid acyl chains. Considering leaflet-specific values, we find that the lipids in contact with PATA-C12 have a lower $\left|S_{\mathrm{CD}}\right|$ values, compared to the lipids in the inner leaflet (see Figure S6), indicating that the lipids in the outer leaflet are more disordered and their acyl chains are more flexible. The effect of PATA-C12 on the diffusion of lipids is rather small. Diffusion coefficients of the PATA-C12 molecules decrease for $d_{\text {unit/POPC }} \geq 0.21$, while the mobility of POPC molecules does not change as $d_{\text {unit/POPC }}$ increases. Therefore, lateral diffusion of lipids cannot account for the trend in membrane fluidity, and we suggest that the increased fluidity of the membrane is primarily due to increased disorder of the lipid chains, resulting from the presence of PATA-C12 molecules.

PATA-C12 Adsorbed on the Membrane Induces an Electrostatic Field. In addition to modifying the bilayer structure, strong adsorption of PATA-C12 molecules to the "outer" leaflet of the POPC membrane leads to an asymmetric distribution of charges in the lipid membrane, hence generating an electrostatic potential difference within the lipid membrane. This potential difference appears due to preferential localization of chloride counterions in the bulk water phase (Figure 2), leaving uncompensated positive charge at the "outer" leaflet of the zwitterionic membrane. The electrostatic field inside the bilayer increases with the increasing density of PATA-C12 units adsorbed on the membrane (Figure 5). We find that the presence of such a voltage between the membrane leaflets favors pore formation (Figure S8 in the Supporting Information). This is consistent with the vast volume of simulation research where membrane pores were induced by the application of an external electric field (electroporation) $)^{55-58}$ or by introducing an ionic charge imbalance at both layers of the membrane. ${ }^{59,60}$ Also note that electroporation is a consequence of the application of an external electric field that acts uniformly on the membrane. In the case of a membrane with adsorbed polycation molecules, the local electric field is created around the polycation molecules and is dependent on the temporal polymer conformations and on the counterion distribution. 
The voltage between the membrane leaflets was below 400 $\mathrm{mV}$ and did not change significantly during the simulation of the M1-M6 systems, in which no spontaneous pore nucleation was observed. In contrast, the interleaflet voltage in the M8 system was initially $\sim 600 \mathrm{mV}$ and decreased to $\sim 300 \mathrm{mV}$ after multiple pores nucleated (Figure 5B). The discharge of the interleaflet voltage was associated with the diffusion of the polymer chains and counterions into the membrane pores (Figure 3). For the M8 system, the initial interleaflet voltage is comparable to the transmembrane potential required for electroporation of doped membranes, as shown in the experiments; for example, Portet and Dimova demonstrated that electric pulses inducing $\sim 700$ $\mathrm{mV}$ transmembrane voltages were able to successfully porate cholesterol-containing dioleoylphosphatidylcholine liposomes, but did not appear to induce pores in pure membranes. ${ }^{61}$

We note that our estimated interleaflet voltages are much lower than the transmembrane voltages used in the MD simulations modeling the electroporation process in pure lipid membranes, where the latter are typically well above $2 \mathrm{~V}{ }^{47}$ Thinner membranes require lower transmembrane voltages to undergo electroporation, ${ }^{47}$ and we found that the adsorption of PATA-C12 also leads to thinning of the membrane. Therefore, we suggest that the voltage between the membrane leaflets and membrane thinning, both effects induced by the asymmetric adhesion of PATA-C12 on the membrane, facilitate the formation of membrane pores.

Membrane Pores in the Presence of PATA-C12. Our experiments show that the adsorption of PATA-C12 on the POPC liposomes strongly increases the permeability of the lipid membrane for polar compounds, even at very low polycation concentrations, suggesting the formation of PATA-C12-induced defects/pores that facilitate the diffusion of polar compounds across the membrane. To check this possibility, we applied direct microscopic visualization of the supported POPC bilayer treated with PATA-C12. AFM observation of the POPC membranes before and after the introduction of the polycation showed that the nanoscale holes were formed in the previously intact areas of the membrane (Figure 11). Poration of supported zwitterionic lipid bilayers by various polycations has been demonstrated using AFM microscopy in several studies. ${ }^{7,62}$ Therefore, we believe that the enhanced permeability of the POPC liposomes decorated with PATA-C12 for hydrophilic compounds is caused by the creation of pores (holes) in the membrane.

The formation of PATA-C12-induced defects/pores is further supported by the spontaneous rupture of the membrane within first tens of nanoseconds of MD simulations for the highest

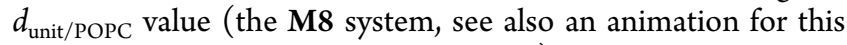
system in the Supporting Information). For lower $d_{\text {unit/POPC }}$ values, we do not observe spontaneous pore formation in our MD simulations; however, the reduction of $\Delta G_{\text {pore }}$ indicates an increased propensity to nucleate pores with increasing contents of the PATA-C12 units. Therefore, we postulate that, at a critical density of polycation units adsorbed on the lipid bilayer $\left(d_{\text {unit/POPC }} \approx 0.8\right)$, there is a rapid nucleation of transmembrane pores in the lipid vesicle. Such a critical density of the PATA$\mathrm{C} 12$ units can be achieved even at low polycation concentrations, since the polycation is nonuniformly absorbed on the membrane surface. In addition, we note two details: (a) careful preparation of initial configurations that allow the PATA-C12 molecules to self-embed without removal of lipids rules out the formation of spontaneous membrane defect/pores at low PATA-C12 concentrations, and (b) the hydrophobic inter- actions (between the polymer dodecyl groups and the lipid acyl chains) in the system are strong, maintain the polycations inserted in the membrane, and do not allow them to escape to the other leaflet through the pore within the simulation time scales.

Our calculated PMFs show that the nucleation of pores in the pure POPC membrane is energetically expensive $\left(\Delta G_{\text {pore }} \approx 65\right.$ $\left.\mathrm{kJ} \mathrm{mol}^{-1}\right)$; however, the addition of six PATA-C12 molecules reduces $\Delta G_{\text {pore }}$ by almost $40 \mathrm{~kJ} \mathrm{~mol}^{-1}$. Since polycations prevent exposure of the hydrophobic interior of the membrane to water, the nucleation barrier for the metastable prepore ${ }^{64}$ decreases with the density of polycation units adsorbed on the lipid bilayer. In other words, the presence of polymer molecules also influences the transition state of membrane pores. ${ }^{63}$ Hence, the free-energy profiles for the formation of membrane pores in the presence of PATA-C12 at different concentrations quantitatively establish the concentration-dependent membrane activity of the polymer.

The pore size was estimated for the M8 system, in which we observed spontaneous pore formation in the bilayer at the early stage of simulation (Figure S4). Because of the dynamic behavior of the lipids and PATA-C12 molecules, the pore dimension was calculated as the averaged distance between the phosphorus atoms of the lipid headgroups facing each other around the pore rim. The diameter of pore induced by PATA$\mathrm{C} 12$ was estimated to be $3.14 \pm 0.09 \mathrm{~nm}$. This result confirms that the polycation is capable of forming pores with sizes that allow the passage of calcein molecules through the bilayer as was experimentally observed for the CL liposomes treated with PATA-C12. In addition, the formation of the toroidal transmembrane pore is accompanied by membrane reorganization (Figures 6 and Figure S4). POPC molecules reorient their hydrophilic headgroups toward the water channel, creating an edge that protects the nonpolar medium of the POPC acyl chains from unfavorable interactions with the aqueous phase.

We used the water-density and slice reaction coordinates to calculate the free energies of pore formation, and we notice some differences between these two methods. Unlike the waterdensity reaction coordinate, the slice coordinate reveals the nucleation barrier for pore opening. Essentially, this nucleation barrier was integrated out in the PMFs calculated using the water-density reaction coordinate, because the system crosses the barrier in a direction orthogonal to the reaction coordinate. ${ }^{19,33}$ Next, the free energies calculated from the slice coordinate for different $N$ converged within $50 \mathrm{~ns}$. The $x-y$ position (in the membrane plane) of the membrane-spanning cylinder in the slice method is not fixed, but instead is dynamically defined, depending on the position of the aqueous defect. ${ }^{33}$ This technical detail avoids movement of the system along the free-energy path (or reaction coordinate) by shifting the defect laterally out of the cylinder, which was identified as a source for hysteresis between pore-opening and pore-closing pathways in the water-density coordinate and convergence issues. ${ }^{19,33}$ Furthermore, because of this missing technical detail, the water-density coordinate also reveals a non-negative $\Delta G_{\text {pore }}$ for spontaneous membrane pores $(N=8$, orange curve in Figure 7). Hence, $\Delta G_{\text {pore }}$ calculated using the slice coordinate for different polycation concentrations (shown in Table 3) are converged and therefore provide a reliable quantitative comparison.

We note that $\Delta G_{\text {pore }}$ is the energy cost of incorporating water molecules into the hydrophobic membrane core and reorganization of lipids around the pore. In other words, the POPC 
molecules are reorganized in such a way that the hydrophilic headgroups protect the nonpolar POPC acyl chains from unfavorable interactions with water. In fact, it would be interesting to further decompose the elastic energy contributions due to the bending of the lipid acyl chains in the presence of a different amount of polycations molecules.

Mechanism of Polycation-Induced Pore Formation. We show that the ability of PATA-C12 to nucleate pores upon asymmetric adsorption on the zwitterionic membranes can be attributed to two effects: (a) polycation-induced electrostatic potential difference between the membrane leaflets, and (b) changes in the membrane structure. At a high density of polymer units adsorbed at a single membrane leaflet, the highly charged polymer generates an interleaflet voltage that is comparable to that applied in electroporation experiments performed for doped membranes. In addition, the adsorbed polycation decreases the packing of the lipid molecules in the bilayer (APL increased) and increases the disordering of the lipid acyl chains (order parameters decreased), thus reducing the membrane thickness and increasing its fluidity in a concentration-dependent manner. As a result, the penetration of water into the membrane and subsequent formation of a water column across the membrane are facilitated. This process is accompanied by reorganization of the membrane, such that the neighboring POPC molecules reorient their headgroups toward the water channel. This new organization of POPC hydrophilic headgroups creates a lining protecting the nonpolar medium of POPC acyl chains from unfavorable interactions with the water phase, thus contributing to the energetic stabilization of the system. The PMFs indicate that $\Delta G_{\text {pore }}$ decreases with increasing density of PATA-C12 units on the membrane surface. Our findings are consistent with the results of $\mathrm{MD}$ simulations for pure bilayers consisting of lipids of various lengths of hydrocarbon chains, which showed that the thinner bilayers are characterized by much lower values of $\Delta G_{\text {pore }}{ }^{26}$

\section{CONCLUSIONS}

We combined comprehensive monolayer and fluorescence experiments and AFM observations with all-atom MD simulations and free-energy calculations to quantitatively investigate the behavior of the hydrophobically modified polycation on the surface of the zwitterionic membrane, used as a protein-free model for cell membranes. We find that PATAC12 strongly adheres to the lipid bilayer with its alkyl chains deeply buried in the hydrophobic core of the membrane. The polycation adhesion on the outer leaflet of the zwitterionic membrane considerably affects the membrane properties. In particular, the asymmetrically adsorbed PATA-C12 generates an electrostatic potential difference between the membrane leaflets, reduces the thickness of the membrane, and increases its fluidity, which is associated with increased disorder in the acyl chains of the lipid molecules. As a result, the free energy for pore nucleation (the energy cost of incorporation of water molecules into the bilayer and reorganization of lipids around the water defects) in the presence of PATA-C12 is strongly reduced, compared to the pure POPC membrane. The extent of these changes is strongly dependent on the density of polycation units adsorbed on the membrane surface, in other words, related to the polymer concentration in the system. The free-energy profiles show that increasing the density of the polycation on the POPC membrane decreases the nucleation barrier for pore opening and also the free energy of pore formation. This suggests that increasing polycation concentration increases the propensity for the nucleation of stable membrane pores in a POPC bilayer.

In conclusion, we have shown that the ability of the hydrophobically modified polycations to form pores in zwitterionic membranes can be attributed to the appearance of the electrostatic field across the bilayer and to the polycationinduced changes in the bilayer organization, such as decreased packing, reduced membrane thickness, and increased disorder of the acyl chains. Such electrostatic potential difference and bilayer reorganization facilitate the penetration of water into the membrane and the formation of defects and pores, as illustrated by the free-energy calculations. Our work provides a quantitative insight into the underlying energetics and molecular mechanism of polycation-induced pore formation in zwitterionic membranes. These results can be of general importance for understanding polycation-enhanced transport of materials through the cell membranes and toxicity of polymers used in biological applications.

\section{ASSOCIATED CONTENT}

\section{Supporting Information}

The Supporting Information is available free of charge on the ACS Publications website at DOI: 10.1021/acsbiomaterials.8b01495.

Additional figures (Figures S1-S10) and tables (Tables S1-S3) (PDF)

Four animations made from the trajectories for systems M1 and M8 (in mpg format) (ZIP)

\section{AUTHOR INFORMATION}

\section{Corresponding Authors}

*Tel.: +49 (0)551 39 14296. Fax: +49 (0)551 39 14082. E-mail: nawasth@gwdg.de (N. Awasthi).

*Tel.: +48 12 6862532. Fax: +48 12 6862750. E-mail: kepczyns@chemia.uj.edu.pl (M. Kepczynski).

ORCID $\odot$

Neha Awasthi: 0000-0003-2034-2705

Jochen S. Hub: 0000-0001-7716-1767

Mariusz Kepczynski: 0000-0002-7304-6881

Present Address

${ }^{\nabla}$ Computational Biomolecular Dynamics Group, Max Planck Institute for Biophysical Chemistry, Am Fassberg 11, 37077 Göttingen, Germany. E-mail: wkopec@mpibpc.mpg.de.

\section{Author Contributions}

The manuscript was written through contributions of all authors. All authors have given approval to the final version of the manuscript.

\section{Notes}

The authors declare no competing financial interest.

\section{ACKNOWLEDGMENTS}

The project was financed by the National Science Centre, Poland (Grant No. 2016/21/B/ST5/00250). N.A. was supported by the Dorothea Schloezer Research Fellowship of the Georg August University, Goettingen. W.K. was supported by the German Research Foundation DFG through No. FOR 2518 "DynIon". N.W. acknowledges the National Science Centre, Poland (NCN) for financial support in the form of ETIUDA doctoral scholarship, on the basis of Decision No. DEC-2017/24/T/ST5/00383. 


\section{REFERENCES}

(1) Rossi, G.; Monticelli, L. Simulating the Interaction of Lipid Membranes with Polymer and Ligand-Coated Nanoparticles. Adv. Phys.: X 2016, 1 (2), 276-296.

(2) Schulz, M.; Olubummo, A.; Binder, W. H. Beyond the LipidBilayer: Interaction of Polymers and Nanoparticles with Membranes. Soft Matter 2012, 8 (18), 4849-4864.

(3) Sikor, M.; Sabin, J.; Keyvanloo, A.; Schneider, M. F.; Thewalt, J. L.; Bailey, A. E.; Frisken, B. J. Interaction of a Charged Polymer with Zwitterionic Lipid Vesicles. Langmuir 2010, 26 (6), 4095-4102.

(4) Yaroslavov, A. A.; Sitnikova, T. A.; Rakhnyanskaya, A. A.; Yaroslavova, E. G.; Sybachin, A. V.; Melik-Nubarov, N. S.; Khomutov, G. B. Variable and low-toxic polyampholytes: Complexation with Biological Membranes. Colloid Polym. Sci. 2017, 295 (8), 1405-1417.

(5) Ding, L.; Chi, E. Y.; Schanze, K. S.; Lopez, G. P.; Whitten, D. G. Insight into the Mechanism of Antimicrobial Conjugated Polyelectrolytes: Lipid Headgroup Charge and Membrane Fluidity Effects. Langmuir 2010, 26 (8), 5544-5550.

(6) Quemeneur, F.; Rinaudo, M.; Pepin-Donat, B. Influence of Polyelectrolyte Chemical Structure on their Interaction with Lipid Membrane of Zwitterionic Liposomes. Biomacromolecules 2008, 9 (8), 2237-2243.

(7) Hong, S.; Leroueil, P. R.; Janus, E. K.; Peters, J. L.; Kober, M.-M.; Islam, M. T.; Orr, B. G.; Baker, J. R., Jr.; Banaszak Holl, M. M. Interaction of Polycationic Polymers with Supported Lipid Bilayers and Cells: Nanoscale Hole Formation and Enhanced Membrane Permeability. Bioconjugate Chem. 2006, 17 (3), 728-734.

(8) Sovadinova, I.; Palermo, E. F.; Huang, R.; Thoma, L. M.; Kuroda, K. Mechanism of Polymer-Induced Hemolysis: Nanosized Pore Formation and Osmotic Lysis. Biomacromolecules 2011, 12 (1), 260268.

(9) Zhang, Z. Y.; Smith, B. D. High-Generation Polycationic Dendrimers Are Unusually Effective at Disrupting Anionic Vesicles: Membrane Bending Model. Bioconjugate Chem. 2000, 11 (6), 805-814. (10) Mecke, A.; Majoros, I. J.; Patri, A. K.; Baker, J. R., Jr.; Banaszak Holl, M. M.; Orr, B. G. Lipid Bilayer Disruption by Polycationic Polymers: The Roles of Size and Chemical Functional Group. Langmuir 2005, 21 (23), 10348-10354.

(11) Eren, T.; Som, A.; Rennie, J. R.; Nelson, C. F.; Urgina, Y.; Nusslein, K.; Coughlin, E. B.; Tew, G. N. Antibacterial and Hemolytic Activities of Quaternary Pyridinium Functionalized Polynorbornenes. Macromol. Chem. Phys. 2008, 209 (5), 516-524.

(12) Wang, H.; Shi, X.; Yu, D.; Zhang, J.; Yang, G.; Cui, Y.; Sun, K.; Wang, J.; Yan, H. Antibacterial Activity of Geminized Amphiphilic Cationic Homopolymers. Langmuir 2015, 31 (50), 13469-13477.

(13) Ivanov, I.; Vemparala, S.; Pophristic, V.; Kuroda, K.; DeGrado, W. F.; McCammon, J. A.; Klein, M. L. Characterization of Nonbiological Antimicrobial Polymers in Aqueous Solution and at Water-Lipid Interfaces from All-Atom Molecular Dynamics. J. Am. Chem. Soc. 2006, 128 (6), 1778-1779.

(14) Kepczynski, M.; Jamroz, D.; Wytrwal, M.; Bednar, J.; Rzad, E.; Nowakowska, M. Interactions of a Hydrophobically Modified Polycation with Zwitterionic Lipid Membranes. Langmuir 2012, 28 (1), 676688.

(15) Smistad, G.; Nyström, B.; Zhu, K.; Grønvold, M. K.; RøvJohnsen, A.; Hiorth, M. Liposomes Coated with Hydrophobically Modified Hydroxyethyl Cellulose: Influence of Hydrophobic Chain Length and Degree of Modification. Colloids Surf., B 2017, 156, 79-86.

(16) Wilkosz, N.; Jamroz, D.; Kopec, W.; Nakai, K.; Yusa, S.; WytrwalSarna, M.; Bednar, J.; Nowakowska, M.; Kepczynski, M. Effect of Polycation Structure on Interaction with Lipid Membranes. J. Phys. Chem. B 2017, 121 (30), 7318-7326.

(17) Ramezanpour, M.; Leung, S. S. W.; Delgado-Magnero, K. H.; Bashe, B. Y. M.; Thewalt, J.; Tieleman, D. P. Computational and Experimental Approaches for Investigating Nanoparticle-Based Drug Delivery Systems. Biochim. Biophys. Acta, Biomembr., Part B 2016, 1858 (7), 1688-1709.

(18) Kostritskii, A. Y.; Kondinskaia, D. A.; Nesterenko, A. M.; Gurtovenko, A. A. Adsorption of Synthetic Cationic Polymers on
Model Phospholipid Membranes: Insight from Atomic-Scale Molecular Dynamics Simulations. Langmuir 2016, 32 (40), 10402-10414.

(19) Awasthi, N.; Hub, J. S. Simulations of Pore Formation in Lipid Membranes: Reaction Coordinates, Convergence, Hysteresis, and Finite-Size Effects. J. Chem. Theory Comput. 2016, 12 (7), 3261-3269.

(20) Kumar, S.; Rosenberg, M. J.; Bouzida, D.; Swendsen, H. R.; Kollman, A. P. THE Weighted Histogram Analysis Method for FreeEnergy Calculations on Biomolecules. I. The Method. J. Comput. Chem. 1992, 13 (8), 1011-1021.

(21) Roux, B. The Calculation of the Potential of Mean Force Using Computer Simulations. Comput. Phys. Commun. 1995, 91 (1-3), 275282.

(22) Roux, B.; Allen, T.; Bernche, S.; Im, W. Theoretical and Computational Models of Biological Ion Channels. Q. Rev. Biophys. 1999, 37 (1), 15-103.

(23) Wohlert, J.; den Otter, W. K.; Edholm, O.; Briels, W. J. Free Energy of a Trans-Membrane Pore Calculated from Atomistic Molecular Dynamics Simulations. J. Chem. Phys. 2006, 124, 154905.

(24) Tolpekina, T. V.; den Otter, W. K.; Briels, W. J. Nucleation Free Energy of Pore Formation in an Amphiphilic Bilayer Studied by Molecular Dynamics Simulations. J. Chem. Phys. 2004, 121, 12060.

(25) Mirjalili, V.; Feig, M. Density-Biased Sampling: A Robust Computational Method for Studying Pore Formation in Membranes. J. Chem. Theory Comput. 2015, 11 (1), 343-350.

(26) Bennett, W. D.; Sapay, N.; Tieleman, D. P. Atomistic Simulations of Pore Formation and Closure in Lipid Bilayers. Biophys. J. 2014, 106 (1), 210-219.

(27) Nagle, J. F.; Tristram-Nagle, S. Structure of Lipid Bilayers. Biochim. Biophys. Acta, Rev. Biomembr. 2000, 1469 (3), 159-195.

(28) Lewandowska, J.; Kepczynski, M.; Bednar, J.; Rząd, E.; Moravcikova, V.; Jachimska, B.; Nowakowska, M. Silicone-Stabilized Liposomes. Colloid Polym. Sci. 2010, 288 (1), 37-45.

(29) Kwolek, U.; Kulig, W.; Wydro, P.; Nowakowska, M.; Róg, T.; Kepczynski, M. Effect of Phosphatidic Acid on Biomembrane: Experimental and Molecular Dynamics Simulations Study. J. Phys. Chem. B 2015, 119 (31), 10042-10051.

(30) Buchoux, S. FATSLiM: A Fast and Robust Software to Analyze MD Simulations of Membranes. Bioinformatics 2017, 33 (1), 133-134.

(31) Stępniewski, M.; Kepczynski, M.; Jamroz, D.; Nowakowska, M.; Rissanen, S.; Vattulainen, I.; Róg, T. Interaction of Hematoporphyrin with Lipid Membranes. J. Phys. Chem. B 2012, 116 (16), 4889-4897.

(32) Hub, J. S.; de Groot, B. L.; van der Spoel, D. G_Wham-A Free Weighted Histogram Analysis Implementation Including Robust Error and Autocorrelation Estimates. J. Chem. Theory Comput. 2010, 6 (12), $3713-3720$

(33) Hub, J. S.; Awasthi, N. Probing a Continuous Polar Defect: A Reaction Coordinate for Pore Formation in Lipid Membranes. J. Chem. Theory Comput. 2017, 13 (5), 2352-2366.

(34) Berger, O.; Edholm, O.; Jahnig, F. Molecular Dynamics Simulations of a Fluid Bilayer of Dipalmitoylphosphatidylcholine at Full Hydration, Constant Pressure, and Constant Temperature. Biophys. J. 1997, 72 (5), 2002-2013.

(35) Bachar, M.; Brunelle, P.; Tieleman, D. P.; Rauk, A. Molecular Dynamics Simulation of a Polyunsaturated Lipid Bilayer Susceptible to Lipid Peroxidation. J. Phys. Chem. B 2004, 108 (22), 7170-7179.

(36) Berendsen, H. J. C.; Grigera, J. R.; Straatsma, T. P. The Missing Term in Effective Pair Potentials. J. Phys. Chem. 1987, 91 (24), 62696271.

(37) Aqvist, J. Ion-Water Interaction Potentials Derived from Free Energy Perturbation Simulations. J. Phys. Chem. 1990, 94 (21), 80218024.

(38) Van der Spoel, D.; Lindahl, E.; Hess, B.; Groenhof, G.; Mark, A. E.; Berendsen, H. J. C. GROMACS: Fast, Flexible, and Free. J. Comput. Chem. 2005, 26 (16), 1701-1718.

(39) Abraham, M. J.; Murtola, T.; Schulz, R.; Páll, S.; Smith, J. C.; Hess, B.; Lindahl, E. GROMACS: High Performance Molecular Simulations Through Multi-Level Parallelism from Laptops to Supercomputers. SoftwareX 2015, 1-2, 19-25. 
(40) Miyamoto, S.; Kollman, P. A. Settle: An Analytical Version of the SHAKE and RATTLE Algorithm for Rigid Water Models. J. Comput. Chem. 1992, 13 (8), 952-962.

(41) Hess, B.; Bekker, H.; Berendsen, H. J. C.; Fraaije, J. G. E. M. LINCS: A linear Constraint Solver for Molecular Simulations. J. Comput. Chem. 1997, 18 (12), 1463-1472.

(42) Nosé, S. A Unified Formulation of the Constant Temperature Molecular Dynamics Methods. J. Chem. Phys. 1984, 81 (1), 511-519.

(43) Hoover, W. G. Canonical dynamics: Equilibrium Phase-Space Distributions. Phys. Rev. A: At., Mol., Opt. Phys. 1985, 31 (3), 16951697.

(44) Parrinello, M.; Rahman, A. Polymorphic Transitions in Single Crystals: A New Molecular Dynamics Method. J. Appl. Phys. 1981, 52 (12), 7182-7190.

(45) Darden, T.; York, D.; Pedersen, L. Particle Mesh Ewald: An $\operatorname{Nlog}(\mathrm{N})$ Method for Ewald Sums in Large Systems. J. Chem. Phys. 1993, 98 (12), 10089-10092.

(46) Esteban-Martin, S.; Risselada, H. J.; Salgado, J.; Marrink, S. J. Stability of Asymmetric Lipid Bilayers Assessed by Molecular Dynamics Simulations. J. Am. Chem. Soc. 2009, 131, 15194-15202.

(47) Delemotte, L.; Tarek, M. Molecular dynamics simulations of lipid membrane electroporation. J. Membr. Biol. 2012, 245 (9), 531-543.

(48) Aoki, P. H. B.; Alessio, P.; Rodriguez-Mendez, M. L.; De Saja Saez, J. A.; Constantino, C. J. L. Taking Advantage of Electrostatic Interactions To Grow Langmuir-Blodgett Films Containing Multilayers of the Phospholipid Dipalmitoylphosphatidylglycerol. Langmuir 2009, 25 (22), 13062-13070.

(49) Kwolek, U.; Jamroz, D.; Janiczek, M.; Nowakowska, M.; Wydro, P.; Kepczynski, M. Interactions of Polyethylenimines with Zwitterionic and Anionic Lipid Membranes. Langmuir 2016, 32 (19), 5004-5018.

(50) Liu, Z.; Zhang, Z.; Zhou, C.; Jiao, Y. Hydrophobic modifications of cationic polymers for gene delivery. Prog. Polym. Sci. 2010, 35 (9), $1144-1162$

(51) Wytrwal, M.; Sarna, M.; Bednar, J.; Kozik, B.; Nowakowska, M.; Kepczynski, M. Formation of Micelles by Hydrophobically Modified Poly(allylamine hydrochloride). Pol. J. Appl. Chem. 2011, 55 (1), 1117.

(52) Shimanouchi, T.; Ishii, H.; Yoshimoto, N.; Umakoshi, H.; Kuboi, R. Calcein Permeation Across Phosphatidylcholine Bilayer Membrane: Effects of Membrane Fluidity, Liposome Size, and Immobilization. Colloids Surf., B 2009, 73 (1), 156-160.

(53) Matsuzaki, K.; Yoneyama, S.; Miyajima, K. Pore Formation and Translocation of Melittin. Biophys. J. 1997, 73 (2), 831-838.

(54) Shao, Z.; Mou, J.; Czajkowsky, D. M.; Yang, J.; Yuan, J. Biological atomic force microscopy: what is achieved and what is needed. $A d v$. Phys. 1996, 45, 1-86.

(55) Tieleman, D. P.; Leontiadou, H.; Mark, A. E.; Marrink, S.-J. Simulation of Pore Formation in Lipid Bilayers by Mechanical Stress and Electric Fields. J. Am. Chem. Soc. 2003, 125 (21), 6382-6383.

(56) Tieleman, D. P. The molecular basis of electroporation. BMC Biochem. 2004, 5, 10.

(57) Tarek, M. Membrane electroporation: a molecular dynamics simulation. Biophys. J. 2005, 88 (6), 4045-4053.

(58) Ziegler, M. J.; Vernier, P. T. Interface water dynamics and porating electric fields for phospholipid bilayers. J. Phys. Chem. B 2008, 112 (43), 13588-13596.

(59) Gurtovenko, A. A.; Vattulainen, I. Pore formation coupled to ion transport through lipid membranes as induced by transmembrane ionic charge imbalance: atomistic molecular dynamics study. J. Am. Chem. Soc. 2005, 127 (50), 17570-17571.

(60) Gurtovenko, A. A.; Vattulainen, I. Ion leakage through transient water pores in protein-free lipid membranes driven by transmembrane ionic charge imbalance. Biophys. J. 2007, 92 (6), 1878-1890.

(61) Portet, T.; Dimova, R. A New Method for Measuring Edge Tensions and Stability of Lipid Bilayers: Effect of Membrane Composition. Biophys. J. 2010, 99 (10), 3264-3273.

(62) Leroueil, P. R.; Berry, S. a.; Duthie, K.; Han, G.; Rotello, V. M.; McNerny, D. Q.; Baker, J. R.; Orr, B. G.; Banaszak Holl, M. M. Wide
Varieties of Cationic Nanoparticles Induce Defects in Supported Lipid Bilayers. Nano Lett. 2008, 8, 420-424.

(63) Ting, C. L.; Awasthi, N.; Muller, M.; Hub, J. S. Metastable Prepores in Tension-Free Lipid Bilayers. Phys. Rev. Lett. 2018, 120 (12), 128103. 